\title{
Quasi-Triangular Spaces, Pompeiu-Hausdorff Quasi-Distances, and Periodic and Fixed Point Theorems of Banach and Nadler Types
}

\author{
Kazimierz Włodarczyk \\ Department of Nonlinear Analysis, Faculty of Mathematics and Computer Science, University of Łódź, Banacha 22, \\ 90-238 Łódź, Poland \\ Correspondence should be addressed to Kazimierz Włodarczyk; wlkzxa@math.uni.lodz.pl
}

Received 19 February 2015; Revised 17 May 2015; Accepted 18 May 2015

Academic Editor: Poom Kumam

Copyright (C) 2015 Kazimierz Włodarczyk. This is an open access article distributed under the Creative Commons Attribution License, which permits unrestricted use, distribution, and reproduction in any medium, provided the original work is properly cited.

Let $C=\left\{C_{\alpha}\right\}_{\alpha \in \mathscr{A}} \in[1 ; \infty)^{\mathscr{A}}, \mathscr{A}$-index set. A quasi-triangular space $\left(X, \mathscr{P}_{C ; \mathscr{A}}\right)$ is a set $X$ with family $\mathscr{P}_{C ; \mathscr{A}}=\left\{p_{\alpha}: X^{2} \rightarrow[0, \infty), \alpha \in\right.$ $\mathscr{A}\}$ satisfying $\forall_{\alpha \in \mathscr{A}} \forall_{u, v, w \in X}\left\{p_{\alpha}(u, w) \leq C_{\alpha}\left[p_{\alpha}(u, v)+p_{\alpha}(v, w)\right]\right\}$. For any $\mathscr{P}_{C ; \mathscr{A}}$, a left (right) family $\mathscr{J}_{C ; \mathscr{A}}$ generated by $\mathscr{P}_{C ; \mathscr{A}}$ is defined to be $\mathscr{J}_{C ; \mathscr{A}}=\left\{J_{\alpha}: X^{2} \rightarrow[0, \infty), \alpha \in \mathscr{A}\right\}$, where $\forall_{\alpha \in \mathscr{A}} \forall_{u, v, w \in X}\left\{J_{\alpha}(u, w) \leq C_{\alpha}\left[J_{\alpha}(u, v)+J_{\alpha}(v, w)\right]\right\}$ and furthermore the property $\forall_{\alpha \in \mathscr{A}}\left\{\lim _{m \rightarrow \infty} p_{\alpha}\left(w_{m}, u_{m}\right)=0\right\}\left(\forall_{\alpha \in \mathscr{A}}\left\{\lim _{m \rightarrow \infty} p_{\alpha}\left(u_{m}, w_{m}\right)=0\right\}\right)$ holds whenever two sequences $\left(u_{m}: m \in \mathbb{N}\right)$ and $\left(w_{m}: m \in \mathbb{N}\right)$ in $X$ satisfy $\forall_{\alpha \in \mathscr{A}}\left\{\lim _{m \rightarrow \infty} \sup _{n>m} J_{\alpha}\left(u_{m}, u_{n}\right)=0\right.$ and $\left.\lim _{m \rightarrow \infty} J_{\alpha}\left(w_{m}, u_{m}\right)=0\right\}\left(\forall_{\alpha \in \mathscr{A}}\left\{\lim _{m \rightarrow \infty} \sup _{n>m} J_{\alpha}\left(u_{n}, u_{m}\right)=0\right.\right.$ and $\left.\left.\lim _{m \rightarrow \infty} J_{\alpha}\left(u_{m}, w_{m}\right)=0\right\}\right)$. In $\left(X, \mathscr{P}_{\mathrm{C} ; \mathscr{A}}\right)$, using the left (right) families $\mathscr{J}_{\mathrm{C} ; \mathscr{A}}$ generated by $\mathscr{P}_{\mathrm{C} ; \mathscr{A}}\left(\mathscr{P}_{\mathrm{C} ; \mathscr{A}}\right.$ is a special case of $\left.\mathscr{J}_{\mathrm{C} ; \mathscr{A}}\right)$, we construct three types of Pompeiu-Hausdorff left (right) quasi-distances on $2^{X}$; for each type we construct of left (right) set-valued quasi-contraction $T: X \rightarrow 2^{X}$, and we prove the convergence, existence, and periodic point theorem for such quasi-contractions. We also construct two types of left (right) single-valued quasi-contractions $T: X \rightarrow X$ and we prove the convergence, existence, approximation, uniqueness, periodic point, and fixed point theorem for such quasi-contractions. $\left(X, \mathscr{P}_{C \cdot d}\right)$ generalize ultra quasitriangular and partiall quasi-triangular spaces (in particular, generalize metric, ultra metric, quasi-metric, ultra quasi-metric, $b$ metric, partial metric, partial $b$-metric, pseudometric, quasi-pseudometric, ultra quasi-pseudometric, partial quasi-pseudometric, topological, uniform, quasi-uniform, gauge, ultra gauge, partial gauge, quasi-gauge, ultra quasi-gauge, and partial quasi-gauge spaces).

\section{Introduction}

The set-valued dynamic system is defined as a pair $(X, T)$, where $X$ is a certain space and $T$ is a set-valued map $T: X \rightarrow$ $2^{X}$; here $2^{X}$ denotes the family of all nonempty subsets of the space $X$. For $m \in\{0\} \cup \mathbb{N}$, we define $T^{[m]}=T \circ T \circ \cdots \circ T(m$ times) and $T^{[0]}=I_{X}$ (an identity map on $\left.X\right)$. By Fix $(T)$ and $\operatorname{Per}(T)$ we denote the sets of all fixed points and periodic points of $T$, respectively; that is, $\operatorname{Fix}(T)=\{w \in X: w \in T(w)\}$ and $\operatorname{Per}(T)=\left\{w \in X: w \in T^{[k]}(w)\right.$ for some $\left.k \in \mathbb{N}\right\}$. A dynamic process or a trajectory starting at $w^{0} \in X$ or a motion of the system $(X, T)$ at $w^{0}$ is a sequence $\left(w^{m}: m \in\{0\} \cup \mathbb{N}\right)$ defined by $w^{m} \in T\left(w^{m-1}\right)$ for $m \in \mathbb{N}$ (see, [1-4]).
Recall that a single-valued dynamic system is defined as a pair $(X, T)$, where $X$ is a certain space and $T$ is a single-valued map $T: X \rightarrow X$; that is, $\forall_{x \in X}\{T(x) \in X\}$. By Fix $(T)$ and $\operatorname{Per}(T)$ we denote the sets of all fixed points and periodic points of $T$, respectively; that is, $\operatorname{Fix}(T)=\{w \in X: w=T(w)\}$ and $\operatorname{Per}(T)=\left\{w \in X: w=T^{[k]}(w)\right.$ for some $\left.k \in \mathbb{N}\right\}$. For each $w^{0} \in X$, a sequence $\left(w^{m}=T^{[m]}\left(w^{0}\right): m \in\{0\} \cup \mathbb{N}\right)$ is called a Picard iteration starting at $w^{0}$ of the system $(X, T)$.

Let $X$ be a (nonempty) set. A distance on $X$ is a map $p$ : $X^{2} \rightarrow[0 ; \infty)$. The set $X$, together with distances on $X$, is called distance spaces.

The following distance spaces are important for several reasons. 
Definition 1. Let $X$ be a (nonempty) set, and let $p: X^{2} \rightarrow$ $[0 ; \infty)$.

(A) $(X, p)$ is called metric if $(\mathrm{i}) \forall_{u, w \in X}\{p(u, w)=0$ iff $u=$ $w\}$, (ii) $\forall_{u, w \in X}\{p(u, w)=p(w, u)\}$, and (iii) $\forall_{u, v, w \in X}\{p(u$, $w) \leq p(u, v)+p(v, w)\}$.

(B) (See [5]) $(X, p)$ is called ultra metric if (i) $\forall_{u, w \in X}$ $\{p(u, w)=0$ iff $u=w\}$, (ii) $\forall_{u, w \in X}\{p(u, w)=p(w, u)\}$, and (iii) $\forall_{u, v, w \in X}\{p(u, w) \leq \max \{p(u, v), p(v, w)\}\}$.

(C) (See $[6,7])(X, p)$ is called $b$-metric with parameter $C \in[1 ; \infty)$ if (i) $\forall_{u, w \in X}\{p(u, w)=0$ iff $u=w\}$, (ii) $\forall_{u, w \in X}$ $\{p(u, w)=p(w, u)\}$, and (iii) $\forall_{u, v, w \in X}\{p(u, w) \leq C[p(u, v)+$ $p(v, w)]\}$.

(D) (See [8]) $(X, p)$ is called partial metric if (i) $\forall_{u, w \in X}$ $\{u=w$ iff $p(u, u)=p(u, w)=p(w, w)\}$, (ii) $\forall_{u, w \in X}\{p(u$, $u) \leq p(u, w)\}$, (iii) $\forall_{u, w \in X}\{p(u, w)=p(w, u)\}$, and (iv) $\forall_{u, v, w \in X}\{p(u, w) \leq p(u, v)+p(v, w)-p(v, v)\}$.

(E) (See $[9])(X, p)$ is called partial $b$-metric with parameter $C \in[1 ; \infty)$ if (i) $\forall_{u, w \in X}\{u=w$ iff $p(u, u)=p(u$, $w)=p(w, w)\}$, (ii) $\forall_{u, w \in X}\{p(u, u) \leq p(u, w)\}$, (iii) $\forall_{u, w \in X}$ $\{p(u, w)=p(w, u)\}$, and (iv) $\forall_{u, v, w \in X}\{p(u, w) \leq C[p(u, v)+$ $p(v, w)]-p(v, v)\}$.

(F) (See [10]) $(X, p)$ is called quasi-metric if (i) $\forall_{u, w \in X}$ $\{p(u, w)=0$ iff $u=w\}$ and (ii) $\forall_{u, v, w \in X}\{p(u, w) \leq p(u, v)+$ $p(v, w)\}$.

(G) $(X, p)$ is called ultra quasi-metric if (i) $\forall_{u, w \in X}\{p(u$, $w)=0$ iff $u=w\}$ and (ii) $\forall_{u, v, w \in X}\{p(u, w) \leq \max \{p(u, v)$, $p(v, w)\}\}$.

(H) The distance $p$ is called pseudometric (or the gauge) on $X$ if (i) $\forall_{u \in X}\{p(u, u)=0\}$, (ii) $\forall_{u, w \in X}\{p(u, w)=p(w, u)\}$, and (iii) $\forall_{u, v, w \in X}\{p(u, w) \leq p(u, v)+p(v, w)\}$.

(I) The distance $p$ is called quasi-pseudometric (or the quasi-gauge) on $X$ if (i) $\forall_{u \in X}\{p(u, u)=0\}$ and (ii) $\forall_{u, v, w \in X}$ $\{p(u, w) \leq p(u, v)+p(v, w)\}$.

(J) (See [11]) The distance $p$ is called ultra quasipseudometric (or the ultra quasi-gauge) on $X$ if (i) $\forall_{u \in X}\{p(u$, $u)=0\}$ and (ii) $\forall_{u, v, w \in X}\{p(u, w) \leq \max \{p(u, v), p(v, w)\}\}$.

Definition 2 (see [12]). Let $X$ be a (nonempty) set, and let $\mathscr{A}$ be an index set.

(A) Each family $\mathscr{D}=\left\{d_{\alpha}: \alpha \in \mathscr{A}\right\}$ of pseudometrics $d_{\alpha}: X^{2} \rightarrow[0, \infty), \alpha \in \mathscr{A}$, is called gauge on $X$. The gauge $\mathscr{D}=\left\{d_{\alpha}: \alpha \in \mathscr{A}\right\}$ on $X$ is called separating if $\forall_{u, w \in X}\{u \neq$ $\left.w \Rightarrow \exists_{\alpha \in \mathscr{A}}\left\{d_{\alpha}(u, w)>0\right\}\right\}$.

(B) Let the family $\mathscr{D}=\left\{d_{\alpha}: \alpha \in \mathscr{A}\right\}$ be separating gauge on $X$. The topology $\mathscr{T}(\mathscr{D})$ having as a subbase the family $\mathscr{B}(\mathscr{D})=\left\{B\left(u, d_{\alpha}, \varepsilon_{\alpha}\right): u \in X, \varepsilon_{\alpha}>0, \alpha \in \mathscr{A}\right\}$ of all balls $B\left(u, d_{\alpha}, \varepsilon_{\alpha}\right)=\left\{v \in X: d_{\alpha}(u, v)<\varepsilon_{\alpha}\right\}$ with $u \in X, \varepsilon_{\alpha}>0$, and $\alpha \in \mathscr{A}$ is called topology induced by $\mathscr{D}$ on $X$; the topology $\mathscr{T}(\mathscr{D})$ is Hausdorff.

(C) A topological space $(X, \mathscr{T})$ such that there is a separating gauge $\mathscr{D}$ on $X$ with $\mathscr{T}=\mathscr{T}(\mathscr{D})$ is called a gauge space and is denoted by $(X, \mathscr{D})$.

Definition 3 (see [13]). Let $X$ be a (nonempty) set, and let $\mathscr{A}$ be an index set.

(A) Each family $\mathscr{P}=\left\{p_{\alpha}, \alpha \in \mathscr{A}\right\}$ of quasi-pseudometrics $p_{\alpha}: X^{2} \rightarrow[0, \infty), \alpha \in \mathscr{A}$, is called quasi-gauge on $X$.
(B) Let the family $\mathscr{P}=\left\{p_{\alpha}: \alpha \in \mathscr{A}\right\}$ be quasi-gauge on $X$. The topology $\mathscr{T}(\mathscr{P})$ having as a subbase of the family $\mathscr{B}(\mathscr{P})=\left\{B\left(u, p_{\alpha}, \varepsilon_{\alpha}\right): u \in X, \varepsilon_{\alpha}>0, \alpha \in \mathscr{A}\right\}$ of all balls $B\left(u, p_{\alpha}, \varepsilon_{\alpha}\right)=\left\{v \in X: p_{\alpha}(u, v)<\varepsilon_{\alpha}\right\}$ with $u \in X, \varepsilon_{\alpha}>0$ and $\alpha \in \mathscr{A}$ is called topology induced by $\mathscr{P}$ on $X$.

(C) A topological space $(X, \mathscr{T})$ such that there is a quasigauge $\mathscr{P}$ on $X$ with $\mathscr{T}=\mathscr{T}(\mathscr{P})$ is called quasi-gauge space and is denoted by $(X, \mathscr{P})$.

Remark 4 (see [13, Theorems 4.2 and 2.6]). Each quasiuniform space and each topological space is the quasi-gauge space.

There is a growing literature concerning set-valued and single-valued dynamic systems in the above defined distance spaces. These studies contain also various extensions of the Banach [14] and Nadler [15, 16] theorems. Of course, there is a huge literature on this topic. For some such spaces and theorems in these spaces, see, for example, M. M. Deza and E. Deza [17], Kirk and Shahzad [18], and references therein.

Recall that the first convergence, existence, approximation, uniqueness, and fixed point result concerning singlevalued contractions in complete metric spaces were obtained by Banach in 1922 [14].

Theorem 5 (see [14]). Let $(X, d)$ be a complete metric space. If $T: X \rightarrow X$ and

$$
\exists_{0 \leq \lambda<1} \forall_{x, y \in X}\{d(T(x), T(y)) \leq \lambda d(x, y)\},
$$

then the following are true: (i)T has a unique fixed point $w$ in $X$ (i.e., there exists $w \in X$ such that $w=T(w)$ and $\operatorname{Fix}(T)=\{w\}$ ); and (ii) for each $w^{0} \in X$, the sequence $\left(T^{[m]}\left(w^{0}\right): m \in \mathbb{N}\right)$ converges to $w$.

The Pompeiu-Hausdorff metric $H^{d}$ on the class of all nonempty closed and bounded subsets $\mathscr{C} \mathscr{B}(X)$ of the metric space $(X, d)$ is defined as follows:

$$
\begin{array}{r}
H^{d}(U, W)=\max \left\{\sup _{u \in U} d(u, W), \sup _{w \in W} d(w, U)\right\}, \\
U, W \in \mathscr{C} \mathscr{B}(X),
\end{array}
$$

where for each $x \in X$ and $V \in \mathscr{C} \mathscr{B}(X), d(x, V)=$ $\inf _{v \in V} d(x, v)$. Using Pompeiu-Hausdorff metric new contractions were received by Nadler in 1967 and 1969 [15, 16] as a tool to study the existence of fixed points of set-valued maps in complete metric spaces.

Theorem 6 (see [15], [16, Theorem 5]). Let $(X, d)$ be a complete metric space. If $T: X \rightarrow \mathscr{C} \mathscr{B}(X)$ and

$$
\exists_{\lambda \in[0 ; 1)} \forall_{x, y \in X}\left\{H^{d}(T(x), T(y)) \leq \lambda d(x, y)\right\},
$$

then $\operatorname{Fix}(T) \neq \varnothing$ (i.e., there exists $w \in X$ such that $w \in T(w)$ ).

Markin $[19,20]$ gave a slighty defferent version of Theorem 6.

Our primary interest is to construct new very general distance spaces, deliver new contractive set-valued and singlevalued dynamic systems in these distance spaces, present 
the new global methods for studying of these dynamic systems in these spaces, and prove new convergence, approximation, existence, uniqueness, periodic point, and fixed point theorems for such dynamic systems.

The goal of the present paper is to introduce and describe the quasi-triangular spaces $\left(X, \mathscr{P}_{\mathrm{C}: \mathcal{A}}\right)$ (Section 2$)$ and more general quasi-triangular spaces $\left(X, \mathscr{P}_{C ; \mathscr{A}}\right)$ with left (right) families $\mathscr{J}_{C ; \mathscr{A}}$ generated by $\mathscr{P}_{C ; \mathscr{A}}$ (Sections 3-5). Moreover, we use new methods and adopt ideas of Pompeiu and Hausdorff (Section 7) (see [21] for an excellent introduction to these ideas), to establish in these spaces some versions of Banach and Nadler theorems (Sections 8 and 9). Here studied dynamic systems are left (right) $\mathscr{J}_{C ; \mathscr{A}^{-}}$admissible or left (right) $\mathscr{P}_{\text {C;A }}$-closed (Section 6). Examples are provided (Sections 1012) and concluding remarks are given (Section 13).

\section{Quasi-Triangular Spaces $\left(X, \mathscr{P}_{C ; \mathscr{A}}\right)$}

It is worth noticing that the distance spaces $\left(X, \mathscr{P}_{C ; \mathscr{A}}\right)$, introduced and described below, are not necessarily topological or Hausdorff or sequentially complete.

Definition 7. Let $X$ be a (nonempty) set, let $\mathscr{A}$ be an index set, and let $C=\left\{C_{\alpha}\right\}_{\alpha \in \mathscr{A}} \in[1 ; \infty)^{\mathscr{A}}$.

(A) One says that a family $\mathscr{P}_{C ; \mathscr{A}}=\left\{p_{\alpha}: X^{2} \rightarrow\right.$ $[0, \infty), \alpha \in \mathscr{A}\}$ of distances is a quasi-triangular family on $X$ if

$$
\forall_{\alpha \in \mathscr{A}} \forall_{u, v, w \in X}\left\{p_{\alpha}(u, w) \leq C_{\alpha}\left[p_{\alpha}(u, v)+p_{\alpha}(v, w)\right]\right\}
$$

A quasi-triangular space $\left(X, \mathscr{P}_{\mathrm{C} ; \mathscr{A}}\right)$ is a set $X$ together with the quasi-triangular family $\mathscr{P}_{C ; \mathscr{A}}$ on $X$.

(B) Let $\left(X, \mathscr{P}_{C ; \mathscr{A}}\right)$ be the quasi-triangular space. One says that $\mathscr{P}_{C ; \mathscr{A}}$ is separating if

$$
\begin{aligned}
\forall_{u, w \in X} & \{u \neq w \\
& \left.\Longrightarrow \exists_{\alpha \in \mathscr{A}}\left\{p_{\alpha}(u, w)>0 \vee p_{\alpha}(w, u)>0\right\}\right\} .
\end{aligned}
$$

(C) If $\left(X, \mathscr{P}_{C ; \mathscr{A}}\right)$ is an quasi-triangular space and $\forall_{\alpha \in \mathscr{A}}$ $\forall_{u, w \in X}\left\{p_{\alpha}^{-1}(u, w)=p_{\alpha}(w, u)\right\}$, then $\forall_{\alpha \in \mathscr{A}} \forall_{u, v, w \in X}\left\{p_{\alpha}^{-1}(u\right.$, $\left.w) \leq C_{\alpha}\left[p_{\alpha}^{-1}(u, v)+p_{\alpha}^{-1}(v, w)\right]\right\}$. One says that the quasitriangular space $\left(X, \mathscr{P}_{C ; \mathscr{A}}^{-1}\right), \mathscr{P}_{C ; \mathscr{A}}^{-1}=\left\{p_{\alpha}^{-1}: X^{2} \rightarrow[0, \infty)\right.$, $\alpha \in \mathscr{A}\}$, is the conjugation of $\left(X, \mathscr{P}_{C ; \mathscr{A}}\right)$.

Remark 8 . In the spaces $\left(X, \mathscr{P}_{C ; \mathscr{A}}\right)$, in general, the distances $p_{\alpha}: X^{2} \rightarrow[0, \infty), \alpha \in \mathscr{A}$, do not vanish on the diagonal; they are asymmetric and do not satisfy triangle inequality (i.e., the properties $\forall_{\alpha \in \mathscr{A}} \forall_{u \in X}\left\{p_{\alpha}(u, u)=0\right\}$ or $\forall_{\alpha \in \mathscr{A}} \forall_{u, w \in X}\left\{p_{\alpha}(u, w)=p_{\alpha}(w, u)\right\}$ or $\forall_{\alpha \in \mathscr{A}} \forall_{u, v, w \in X}\left\{p_{\alpha}(u\right.$, $\left.w) \leq p_{\alpha}(u, v)+p_{\alpha}(v, w)\right\}$ do not necessarily hold); see Section 10 .

Definition 9. Let $X$ be a (nonempty) set, let $\mathscr{A}$ be an index set, and let $C=\left\{C_{\alpha}\right\}_{\alpha \in \mathscr{A}} \in[1 ; \infty)^{\mathscr{A}}$.
(A) One says that a family $\mathscr{L}_{C ; \mathscr{A}}=\left\{l_{\alpha}: X^{2} \rightarrow[0, \infty)\right.$, $\alpha \in \mathscr{A}\}$ of distances on $X$ is a ultra quasi-triangular family if

$$
\begin{aligned}
& \forall_{\alpha \in \mathscr{A}} \forall_{u, v, w \in X}\left\{l_{\alpha}(u, w)\right. \\
& \left.\quad \leq C_{\alpha} \max \left\{l_{\alpha}(u, v), l_{\alpha}(v, w)\right\}\right\} .
\end{aligned}
$$

An ultra quasi-triangular space $\left(X, \mathscr{L}_{C ; \mathscr{A}}\right)$ is a set $X$ together with the ultra quasi-triangular family $\mathscr{L}_{C ; \mathscr{A}}$ on $X$.

(B) One says that a family $\mathcal{S}_{C ; \mathscr{A}}=\left\{s_{\alpha}: X^{2} \rightarrow[0, \infty)\right.$, $\alpha \in \mathscr{A}\}$ of distances on $X$ is a partial quasi-triangular family if

$$
\begin{aligned}
& \forall_{\alpha \in \mathscr{A}} \forall_{u, v, w \in X}\left\{s_{\alpha}(u, w) \leq C_{\alpha}\left[s_{\alpha}(u, v)+s_{\alpha}(v, w)\right]\right. \\
& \left.-s_{\alpha}(v, v)\right\} .
\end{aligned}
$$

A partial quasi-triangular space $\left(X, \mathcal{S}_{C ; \mathscr{A}}\right)$ is a set $X$ together with the partial quasi-triangular family $\delta_{C ; A}$ on $X$.

Remark 10. It is worth noticing that quasi-triangular spaces generalize ultra quasi-triangular and partial quasi-triangular spaces (in particular, generalize metric, ultra metric, quasimetric, ultra quasi-metric, $b$-metric, partial metric, partial $b$-metric, pseudometric, quasi-pseudometric, ultra quasipseudometric, partial quasi-pseudometric, topological, uniform, quasi-uniform, gauge, ultra gauge, partial gauge, quasigauge, ultra quasi-gauge, and partial quasi-gauge spaces).

\section{Left (Right) Families $\mathscr{J}_{\mathrm{C} ; \not / \mathscr{l}}$ Generated by

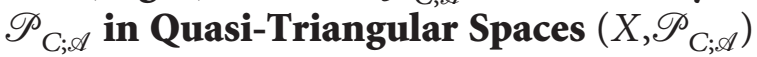

In the metric spaces $(X, d)$ there are several types of distances (determined by $d$ ) which generalize metrics $d$. First these distances were introduced by Tataru [22]. More general concepts of distances in metric spaces $(X, d)$ which generalize $d$, of this sort, are given by Kada et al. [23] ( $w$-distances), Lin and Du [24] ( $\tau$-functions), Suzuki [25] ( $\tau$-distances), and Ume [26] ( $u$-distance). Distances in uniform spaces were given by Vályi [27]. In the appearing literature, these distances and their generalizations in other spaces provide efficient tools to study various problems of fixed point theory; see, for example, [28-30] and references therein. In this paper we also generalize these ideas.

Let $\mathscr{P}_{C ; \mathscr{A}}$ be the quasi-triangular family on $X$. It is natural to define the notions of left (right) families $\mathscr{J}_{C ; \mathscr{A}}$ generated by $\mathscr{P}_{C ; \mathscr{A}}$ which provide new structures on $X$.

Definition 11. Let $\left(X, \mathscr{P}_{C ; \mathscr{A}}\right)$ be the quasi-triangular space.

(A) The family $\mathscr{J}_{C ; \mathscr{A}}=\left\{J_{\alpha}: \alpha \in \mathscr{A}\right\}$ of distances $J_{\alpha}: X^{2} \rightarrow[0, \infty), \alpha \in \mathscr{A}$, is said to be a left (right) family generated by $\mathscr{P}_{C ; \mathscr{A}}$ if

$(\mathscr{J} 1) \forall_{\alpha \in \mathscr{A}} \forall_{u, v, w \in X}\left\{J_{\alpha}(u, w) \leq C_{\alpha}\left[J_{\alpha}(u, v)+J_{\alpha}(v, w)\right]\right\}$; and furthermore. 
$(\mathscr{J} 2)$ For any sequences $\left(u_{m}: m \in \mathbb{N}\right)$ and $\left(w_{m}: m \in \mathbb{N}\right)$ in $X$ satisfying

$$
\begin{gathered}
\forall_{\alpha \in \mathscr{A}}\left\{\lim _{m \rightarrow \infty} \sup _{n>m} J_{\alpha}\left(u_{m}, u_{n}\right)=0\right\}, \\
\left(\forall_{\alpha \in \mathscr{A}}\left\{\lim _{m \rightarrow \infty} \sup _{n>m} J_{\alpha}\left(u_{n}, u_{m}\right)=0\right\}\right), \\
\forall_{\alpha \in \mathscr{A}}\left\{\lim _{m \rightarrow \infty} J_{\alpha}\left(w_{m}, u_{m}\right)=0\right\}, \\
\left(\forall_{\alpha \in \mathscr{A}}\left\{\lim _{m \rightarrow \infty} J_{\alpha}\left(u_{m}, w_{m}\right)=0\right\}\right),
\end{gathered}
$$

the following holds

$$
\begin{gathered}
\forall_{\alpha \in \mathscr{A}}\left\{\lim _{m \rightarrow \infty} p_{\alpha}\left(w_{m}, u_{m}\right)=0\right\}, \\
\left(\forall_{\alpha \in \mathscr{A}}\left\{\lim _{m \rightarrow \infty} p_{\alpha}\left(u_{m}, w_{m}\right)=0\right\}\right) .
\end{gathered}
$$

(B) $\mathbb{J}_{\left(X, \mathscr{P}_{C ; \mathscr{A}}\right)}^{L}\left(\rrbracket_{\left(X, \mathscr{P}_{\mathrm{C} ; \mathscr{A}}\right)}^{R}\right)$ is the set of all left (right) families $\mathscr{J}_{\mathrm{C} ; \mathscr{A}}$ on $X$ generated by $\mathscr{P}_{C ; \mathscr{A}}$.

Remark 12. From Definition 11 if follows that $\mathscr{P}_{C ; \mathscr{A}} \in$ $\mathbb{J}_{\left(X, \mathscr{P}_{C ; \mathscr{A})}\right)}^{L} \cap \mathbb{J}_{\left(X, \mathscr{P}_{C ; \mathscr{A}}\right)}^{R}$. Moreover, there are families $\mathscr{J}_{C ; \mathscr{A}} \in$ $\mathbb{J}_{\left(X, \mathscr{P}_{C ; \mathscr{A}}\right)}^{L}$ and $\mathscr{J}_{C ; \mathscr{A}} \in \mathbb{J}_{\left(X, \mathscr{P}_{\mathrm{C} ; \mathcal{A}}\right)}^{R}$ such that the distances $J_{\alpha}, \alpha \in$ $\mathscr{A}$, do not vanish on the diagonal, are asymmetric, and are quasi-triangular and thus are not metric, ultra metric, quasimetric, ultra quasi-metric, $b$-metric, partial metric, partial $b$-metric, pseudometric (gauge), quasi-pseudometric (quasigauge), and ultra quasi-pseudometric (ultra quasi-gauge).

\section{Relations between $\mathscr{J}_{C ; \mathscr{A}}$ and $\mathscr{P}_{C ; \mathscr{A}}$}

Remark 13. The following result shows that Definition 11 is correct and that $\rrbracket_{\left(X, \mathscr{P}_{C ; \mathscr{A}}\right)}^{L} \backslash\left\{\mathscr{P}_{C ; \mathscr{A}}\right\} \neq \varnothing$ and $\rrbracket_{\left(X, \mathscr{P}_{C ; \mathscr{A}}\right)}^{R} \backslash$ $\left\{\mathscr{P}_{C ; \mathscr{A}}\right\} \neq \varnothing$.

Theorem 14. Let $\left(X, \mathscr{P}_{C ; \mathscr{A}}\right)$ be the quasi-triangular space. Let $E \subset X$ be a set containing at least two different points and let $\left\{\mu_{\alpha}\right\}_{\alpha \in \mathscr{A}} \in(0 ; \infty)^{\mathscr{A}}$ where

$$
\begin{gathered}
\forall_{\alpha \in \mathscr{A}}\left\{\mu_{\alpha} \geq \frac{\delta_{\alpha}(E)}{2 C_{\alpha}}\right\}, \\
\forall_{\alpha \in \mathscr{A}}\left\{\delta_{\alpha}(E)=\sup \left\{p_{\alpha}(u, w): u, w \in E\right\}\right\} .
\end{gathered}
$$

If $\mathscr{J}_{\mathrm{C} ; \mathscr{A}}=\left\{J_{\alpha}: \alpha \in \mathscr{A}\right\}$ where, for each $\alpha \in \mathscr{A}$, the distance $J_{\alpha}: X^{2} \rightarrow[0, \infty)$ is defined by

$$
J_{\alpha}(u, w)= \begin{cases}p_{\alpha}(u, w) & \text { if } E \cap\{u, w\}=\{u, w\} \\ \mu_{\alpha} & \text { if } E \cap\{u, w\} \neq\{u, w\},\end{cases}
$$

then $\mathscr{J}_{C ; \mathscr{A}}$ is left and right family generated by $\mathscr{P}_{C ; \mathscr{A}}$.

Proof. Indeed, we see that condition $(\mathscr{J} 1)$ does not hold only if there exist some $\alpha_{0} \in \mathscr{A}$ and $u_{0}, v_{0}, w_{0} \in X$ such that

$$
J_{\alpha_{0}}\left(u_{0}, w_{0}\right)>C_{\alpha_{0}}\left[J_{\alpha_{0}}\left(u_{0}, v_{0}\right)+J_{\alpha_{0}}\left(v_{0}, w_{0}\right)\right] \text {. }
$$

Then (15) implies $\left\{u_{0}, v_{0}, w_{0}\right\} \cap E \neq\left\{u_{0}, v_{0}, w_{0}\right\}$ and the following Cases 1-4 hold.

Case 1. If $\left\{u_{0}, w_{0}\right\} \subset E$, then $v_{0} \notin E$ and, by (16) and (15), $p_{\alpha_{0}}\left(u_{0}, w_{0}\right)>2 C_{\alpha_{0}} \mu_{\alpha_{0}}$. Therefore, by (14), $p_{\alpha_{0}}\left(u_{0}, w_{0}\right)>$ $2 C_{\alpha_{0}} \mu_{\alpha_{0}} \geq \delta_{\alpha_{0}}(E)$. This is impossible.

Case 2. If $u_{0} \in E$ and $w_{0} \notin E$, then (16) and (15) give $\mu_{\alpha_{0}}>$ $C_{\alpha_{0}}\left[p_{\alpha_{0}}\left(u_{0}, v_{0}\right)+\mu_{\alpha_{0}}\right] \geq C_{\alpha_{0}} \mu_{\alpha_{0}}$ whenever $v_{0} \in E$ or $\mu_{\alpha_{0}}>$ $C_{\alpha_{0}}\left[\mu_{\alpha_{0}}+\mu_{\alpha_{0}}\right]=2 C_{\alpha_{0}} \mu_{\alpha_{0}}$ whenever $v_{0} \notin E$. This is impossible.

Case 3. If $u_{0} \notin E$ and $w_{0} \in E$, then (16) and (15) give $\mu_{\alpha_{0}}>$ $C_{\alpha_{0}}\left[\mu_{\alpha_{0}}+p_{\alpha_{0}}\left(v_{0}, w_{0}\right)\right] \geq C_{\alpha_{0}} \mu_{\alpha_{0}}$ whenever $v_{0} \in E$ or $\mu_{\alpha_{0}}>$ $C_{\alpha_{0}}\left[\mu_{\alpha_{0}}+\mu_{\alpha_{0}}\right]=2 C_{\alpha_{0}} \mu_{\alpha_{0}}$ whenever $v_{0} \notin E$. This is impossible.

Case 4. If $u_{0} \notin E$ and $w_{0} \notin E$, then (16) and (15) give $\mu_{\alpha_{0}}>$ $C_{\alpha_{0}}\left[\mu_{\alpha_{0}}+\mu_{\alpha_{0}}\right]=2 C_{\alpha_{0}} \mu_{\alpha_{0}}$ for $v_{0} \in X$. This is impossible.

Therefore, $\forall_{\alpha \in \mathscr{A}} \forall_{u, v, w \in X}\left\{J_{\alpha}(u, w) \leq C_{\alpha}\left[J_{\alpha}(u, v)+J_{\alpha}(v\right.\right.$, $w)]\}$; that is, the condition $(\mathscr{J} 1)$ holds.

Assume now that the sequences $\left(u_{m}: m \in \mathbb{N}\right)$ and $\left(w_{m}:\right.$ $m \in \mathbb{N}$ ) in $X$ satisfy (8) and (10). Then (12) holds. Indeed, (10) implies

$$
\forall_{\alpha \in \mathscr{A}} \forall_{0<\varepsilon<\mu_{\alpha}} \exists_{m_{0}=m_{0}(\alpha) \in \mathbb{N}} \forall_{m \geq m_{0}}\left\{J_{\alpha}\left(w_{m}, u_{m}\right)<\varepsilon\right\} .
$$

Denoting $m^{\prime}=\min \left\{m_{0}(\alpha): \alpha \in \mathscr{A}\right\}$, we see, by (17) and (15), that $\forall_{m \geq m^{\prime}}\left\{E \cap\left\{w_{m}, u_{m}\right\}=\left\{w_{m}, u_{m}\right\}\right\}$. Then, in view of Definition 11(A), (15), and (17), this implies $\forall_{\alpha \in \mathscr{A}} \forall_{0<\varepsilon<\mu_{\alpha}} \exists_{m^{\prime} \in \mathbb{N}} \forall_{m \geq m^{\prime}}\left\{p_{\alpha}\left(w_{m}, u_{m}\right)=J_{\alpha}\left(w_{m}, u_{m}\right)<\varepsilon\right\}$. Hence we obtain that the sequences $\left(u_{m}: m \in \mathbb{N}\right)$ and $\left(w_{m}: m \in \mathbb{N}\right)$ satisfy (12). Thus we see that $\mathscr{J}_{C ; \mathscr{A}}$ is left family generated by $\mathscr{P}_{C ; \mathscr{A}}$.

In a similar way, we show that (13) holds if $\left(u_{m}: m \in \mathbb{N}\right)$ and $\left(w_{m}: m \in \mathbb{N}\right)$ in $X$ satisfy (9) and (11). Therefore, $\mathscr{J}_{C ; \mathscr{A}}$ is right family generated by $\mathscr{P}_{C ; \mathscr{A}}$. We proved that $\mathscr{J}_{C ; \mathscr{A}} \in$ $\mathbb{J}_{\left(X, \mathscr{P}_{C ; \mathscr{d}}\right)}^{L} \cap \mathbb{J}_{\left(X, \mathscr{P}_{C ; \mathscr{d}}\right)}^{R}$ holds.

The following is interesting in respect to its use.

Theorem 15. Let $\left(X, \mathscr{P}_{C ; \mathscr{A}}\right)$ be the quasi-triangular space, and let $\mathscr{J}_{C ; A}$ be the left (right) family generated by $\mathscr{P}_{C ; \mathscr{A}}$. If $\mathscr{P}_{C ; \mathscr{A}}$ is separating on $X$ (i.e., (5) holds), then $\mathscr{J}_{\mathrm{C} ; \mathcal{A}}$ is separating on $X$; that is,

$$
\begin{aligned}
\forall_{u, w \in X} & \{u \neq w \\
\Longrightarrow & \left.\exists_{\alpha \in \mathscr{A}}\left\{J_{\alpha}(u, w)>0 \vee J_{\alpha}(w, u)>0\right\}\right\}
\end{aligned}
$$

holds.

Proof. We begin by supposing that $u_{0}, w_{0} \in X, u_{0} \neq w_{0}$, and $\forall_{\alpha \in \mathscr{A}}\left\{J_{\alpha}\left(u_{0}, w_{0}\right)=0 \wedge J_{\alpha}\left(w_{0}, u_{0}\right)=0\right\}$. Then $(\mathscr{J} 1)$ implies $\forall_{\alpha \in \mathscr{A}}\left\{J_{\alpha}\left(u_{0}, u_{0}\right) \leq C_{\alpha}\left[J_{\alpha}\left(u_{0}, w_{0}\right)+J_{\alpha}\left(w_{0}, u_{0}\right)\right]=0\right\}$ or, equivalently, $\forall_{\alpha \in \mathscr{A}}\left\{J_{\alpha}\left(u_{0}, u_{0}\right)=J_{\alpha}\left(w_{0}, u_{0}\right)=0\right\}$ and $\forall_{\alpha \in \mathscr{A}}\left\{J_{\alpha}\left(u_{0}\right.\right.$, $\left.\left.u_{0}\right)=J_{\alpha}\left(u_{0}, w_{0}\right)=0\right\}$. Assuming that $u_{m}=u_{0}$ and $w_{m}=w_{0}$, $m \in \mathbb{N}$, we conclude that $\forall_{\alpha \in \mathscr{A}}\left\{\lim _{m \rightarrow \infty} \sup _{n>m} J_{\alpha}\left(u_{m}, u_{n}\right)=\right.$ $\left.\lim _{m \rightarrow \infty} J_{\alpha}\left(w_{m}, u_{m}\right)=0\right\}$ and $\forall_{\alpha \in \mathscr{A}}\left\{\lim _{m \rightarrow \infty} \sup _{n>m} J_{\alpha}\left(u_{n}\right.\right.$, $\left.\left.u_{m}\right)=\lim _{m \rightarrow \infty} J_{\alpha}\left(u_{m}, w_{m}\right)=0\right\}$. Therefore, it is not hard to see that (8)-(11) hold and, by $(\mathscr{J} 2)$, the above 
considerations lead to the following conclusion: $u_{0} \neq w_{0} \wedge$ $\forall_{\alpha \in \mathscr{A}}\left\{\lim _{m \rightarrow \infty} p_{\alpha}\left(w_{m}, u_{m}\right)=\lim _{m \rightarrow \infty} p_{\alpha}\left(u_{m}, w_{m}\right)=0\right\}$ or, equivalently, $u_{0} \neq w_{0} \wedge \forall_{\alpha \in \mathscr{A}}\left\{p_{\alpha}\left(w_{0}, u_{0}\right)=p_{\alpha}\left(u_{0}, w_{0}\right)=\right.$ $0\}$. However, $\mathscr{P}_{C ; \mathscr{A}}$ is separating. A contradiction. Therefore, $\mathscr{J}_{C ; \mathscr{A}}$ is separating.

\section{Left (Right) $\mathscr{J}_{\mathrm{C} ; \mathscr{A}^{-}}$-Convergences and Left (Right) $\mathscr{J}_{C: \mathscr{A}}$-Sequentially Completeness}

Definition 16. Let $\left(X, \mathscr{P}_{C ; \mathscr{A}}\right)$ be the quasi-triangular space, and let $\mathscr{J}_{C ; \mathscr{A}}$ be the left (right) family generated by $\mathscr{P}_{C ; \mathscr{A}}$.

(A) One says that a sequence $\left(u_{m}: m \in \mathbb{N}\right) \subset X$ is left

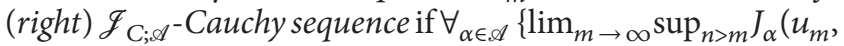
$\left.\left.u_{n}\right)=0\right\}\left(\forall_{\alpha \in \mathscr{A}}\left\{\lim _{m \rightarrow \infty} \sup _{n>m} J_{\alpha}\left(u_{n}, u_{m}\right)=0\right\}\right)$.

(B) Let $u \in X$ and let $\left(u_{m}: m \in \mathbb{N}\right) \subset X$. One says that the sequence $\left(u_{m}: m \in \mathbb{N}\right)$ is left (right) $\mathscr{F}_{C ; \mathscr{A} \text {-convergent to }}$ $u$ if $u \in \operatorname{LIM}_{\left(u_{m}: m \in \mathbb{N}\right)}^{L-\mathscr{F}_{C ; s}} \neq \varnothing\left(u \in \operatorname{LIM}_{\left(u_{m}: m \in \mathbb{N}\right)}^{R-\mathscr{f}_{C ; s}} \neq \varnothing\right)$ where

$$
\begin{aligned}
& \operatorname{LIM}_{\left(u_{m}: m \in \mathbb{N}\right)}^{L-\mathscr{J}_{C ; \mathscr{A}}}=\{x \\
& \left.\quad \in X: \forall_{\alpha \in \mathscr{A}}\left\{\lim _{m \rightarrow \infty} J_{\alpha}\left(x, u_{m}\right)=0\right\}\right\}, \\
& \left(\operatorname{LIM}_{\left(u_{m}: m \in \mathbb{N}\right)}^{R-\mathscr{J}_{C ; \mathscr{A}}}\right. \\
& \left.\quad=\left\{x \in X: \forall_{\alpha \in \mathscr{A}}\left\{\lim _{m \rightarrow \infty} J_{\alpha}\left(u_{m}, x\right)=0\right\}\right\}\right) .
\end{aligned}
$$

(C) One says that a sequence $\left(u_{m}: m \in \mathbb{N}\right) \subset$

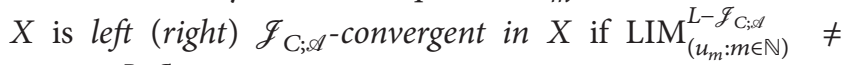
$\varnothing\left(\operatorname{LIM}_{\left(u_{m}: m \in \mathbb{N}\right)}^{R-\mathscr{I}_{C ; A}} \neq \varnothing\right)$.

(D) If every left (right) $\mathscr{J}_{C ; \mathscr{A}}$-Cauchy sequence $\left(u_{m}\right.$ : $m \in \mathbb{N}$ ) $\subset X$ is left (right) $\mathscr{J}_{C ; \mathscr{A}}$-convergent in $X$ (i.e., $\left.\operatorname{LIM}_{\left(u_{m}: m \in \mathbb{N}\right)}^{L-\mathscr{F}_{C ; \mathscr{A}}} \neq \varnothing\left(\operatorname{LIM}_{\left(u_{m}: m \in \mathbb{N}\right)}^{R-\mathscr{F}_{C ; \mathscr{A}}} \neq \varnothing\right)\right)$, then $\left(X, \mathscr{P}_{C ; \mathscr{A}}\right)$ is called left (right) $\mathscr{J}_{\mathrm{C} ; \mathscr{A}^{-}}$-sequentially complete.

Remark 17. The structures on $X$ determined by left (right) families $\mathscr{J}_{C ; \mathscr{A}}$ generated by $\mathscr{P}_{C ; \mathscr{A}}$ are more general than the structure on $X$ determined by $\mathscr{P}_{C ; \mathscr{A}}$; see Remark 34 .

Remark 18. Let $\left(X, \mathscr{P}_{C ; \mathscr{A}}\right)$ be the quasi-triangular space. It is clear that if $\left(u_{m}: m \in \mathbb{N}\right)$ is left (right) $\mathscr{P}_{C ; \mathscr{A}^{-}}$-convergent in $X$, then $\operatorname{LIM}_{\left(u_{m}: m \in \mathbb{N}\right)}^{L-\mathscr{P}_{C ; \mathscr{A}}} \subset \operatorname{LIM}_{\left(v_{m}: m \in \mathbb{N}\right)}^{L-\mathscr{P}_{C ; \mathscr{A}}}\left(\operatorname{LIM}_{\left(u_{m}: m \in \mathbb{N}\right)}^{R-\mathscr{P}_{C ; A}}\right.$ $\left.\operatorname{LIM}_{\left(v_{m}: m \in \mathbb{N}\right)}^{R-\mathscr{P}_{C \cdot A}}\right)$ for each subsequence $\left(v_{m}: m \in \mathbb{N}\right)$ of $\left(u_{m}:\right.$ $m \in \mathbb{N})$.

Definition 19. One says that $\left(X, \mathscr{P}_{C ; \mathscr{A}}\right)$ is left (right) Hausdorff if for each left (right) $\mathscr{P}_{C ; \mathscr{A}}$-convergent in $X$ sequence $\left(u_{m}\right.$ : $m \in \mathbb{N})$ the set $\operatorname{LIM}_{\left(u_{m}: m \in \mathbb{N}\right)}^{L-\mathscr{P}_{C ; \mathscr{d}}}\left(\operatorname{LIM}_{\left(u_{m}: m \in \mathbb{N}\right)}^{R-\mathscr{P}_{C ; \mathscr{d}}}\right)$ is a singleton.

\section{Left (Right) $\mathscr{J}_{C ; \mathscr{A}}$-Admissible and Left (Right) $\mathscr{P}_{C ; \mathscr{A}}$-Closed Set-Valued Maps}

The following terminologies will be much used in the sequel.
Definition 20. Let $\left(X, \mathscr{P}_{C ; \mathscr{A}}\right)$ be the quasi-triangular space, and let $\mathscr{J}_{C ; \mathscr{A}}$ be the left (right) family generated by $\mathscr{P}_{C ; \mathscr{A}}$. Let $(X, T)$ be the set-valued dynamic system, $T: X \rightarrow 2^{X}$.

(A) Given $w^{0} \in X$, One says that $(X, T)$ is left (right)

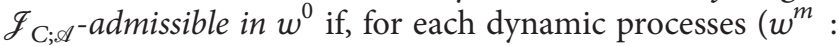
$m \in\{0\} \cup \mathbb{N})$ starting at $w^{0}, \forall_{m \in\{0\} \cup \mathbb{N}}\left\{w^{m+1} \in T\left(w^{m}\right)\right\}$, $\operatorname{LIM}_{\left(w^{m}: m \in\{0\} \cup \mathbb{N}\right)}^{L-\mathscr{I}_{C ; A}} \neq \varnothing\left(\operatorname{LIM}_{\left(w^{m}: m \in\{0\} \cup \mathbb{N}\right)}^{R-\mathscr{F}_{C ; A}} \neq \varnothing\right)$ whenever

$$
\begin{gathered}
\forall_{\alpha \in \mathscr{A}}\left\{\lim _{m \rightarrow \infty} \sup _{n>m} J_{\alpha}\left(w^{m}, w^{n}\right)=0\right\} \\
\left(\forall_{\alpha \in \mathscr{A}}\left\{\lim _{m \rightarrow \infty} \sup _{n>m} J_{\alpha}\left(w^{n}, w^{m}\right)=0\right\}\right) .
\end{gathered}
$$

(B) One says that $(X, T)$ is left (right) $\mathscr{F}_{C ; \mathcal{A}}$-admissible on

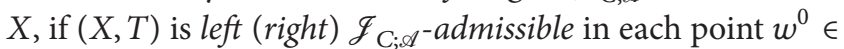
$X$.

Remark 21. Let $\left(X, \mathscr{P}_{C ; \mathscr{A}}\right)$ be the quasi-triangular space and let $\mathscr{J}_{C ; \mathscr{A}}$ be the left (right) family generated by $\mathscr{P}_{C ; \mathscr{A}}$. Let $(X, T)$ be the set-valued dynamic system on $X$. If $\left(X, \mathscr{P}_{C ; \mathscr{A}}\right)$

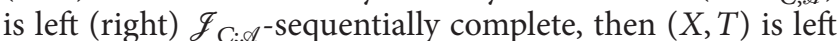
(right) $\mathscr{J}_{C ; \mathscr{A}}$-admissible on $X$ but the converse not necessarily holds.

We can define also the following generalization of continuity.

Definition 22. Let $\left(X, \mathscr{P}_{C ; \mathscr{A}}\right)$ be the quasi-triangular space. Let $(X, T)$ be the set-valued dynamic system, $T: X \rightarrow 2^{X}$, and let $k \in \mathbb{N}$. The set-valued dynamic system $\left(X, T^{[k]}\right)$ is said to be a left (right) $\mathscr{P}_{C ; \mathscr{A}^{-} \text {-closed }} \mathrm{X}$ if for every sequence $\left(x_{m}: m \in \mathbb{N}\right)$ in $T^{[k]}(X)$, left (right) $\mathscr{P}_{C ; \mathscr{A}^{-} \text {-converging in }}$ $X$ (thus $\left.\operatorname{LIM}_{\left(x_{m}: m \in \mathbb{N}\right)}^{L-\mathscr{P}_{C ; A}} \neq \varnothing\left(\operatorname{LIM}_{\left(x_{m}: m \in \mathbb{N}\right)}^{R-\mathscr{P}_{C, \mathscr{A}}} \neq \varnothing\right)\right)$ and having subsequences $\left(v_{m}: m \in \mathbb{N}\right)$ and $\left(u_{m}: m \in \mathbb{N}\right)$ satisfying $\forall_{m \in \mathbb{N}}\left\{v_{m} \in T^{[k]}\left(u_{m}\right)\right\}$, the following property holds: there exists $x \in \operatorname{LIM}_{\left(x_{m}: m \in \mathbb{N}\right)}^{L-\mathscr{P}_{C ;}}\left(x \in \operatorname{LIM}_{\left(x_{m}: m \in \mathbb{N}\right)}^{R-\mathscr{P}_{C ;}}\right)$ such that $x \in$ $T^{[k]}(x)\left(x \in T^{[k]}(x)\right)$.

\section{Left (Right) Pompeiu-Hausdorff Quasi-Distances and Left (Right) Set-Valued Quasi-Contractions}

In this section, in the quasi-triangular spaces $\left(X, \mathscr{P}_{C ; \mathscr{A}}\right)$, using left (right) families $\mathscr{F}_{C: \mathscr{A}}$ generated by $\mathscr{P}_{\text {C:AdA }}$, we define three types of left (right) Pompeiu-Hausdorff quasi-distances on $2^{X}$, and for each type a left (right) set-valued quasicontraction $T: X \rightarrow 2^{X}$ is constructed.

Definition 23. Let $\left(X, \mathscr{P}_{C ; \mathscr{A}}\right)$ be the quasi-triangular space, and let $\mathscr{J}_{C ; \mathscr{A}}$ be the left (right) family generated by $\mathscr{P}_{C ; \mathscr{A}}$. Let $\lambda=\left\{\lambda_{\alpha}\right\}_{\alpha \in \mathscr{A}} \in[0 ; 1)^{\mathscr{A}}$, let $(X, T)$ be a set-valued dynamic system, $T: X \rightarrow 2^{X}$, and let $\eta \in\{1,2,3\}$. Let

$$
\begin{gathered}
\forall_{\alpha \in \mathscr{A}} \forall_{x \in X} \forall_{V \in 2^{X}} \quad\left\{J_{\alpha}(x, V)=\inf \left\{J_{\alpha}(x, v): v \in V\right\}\right. \\
\left.\wedge J_{\alpha}(V, x)=\inf \left\{J_{\alpha}(v, x): v \in V\right\}\right\} .
\end{gathered}
$$


(A) Let $\mathscr{J}_{C ; \mathscr{A}} \in \rrbracket_{\left(X, \mathscr{P}_{C ; A}\right)}^{L}$. If

$$
\begin{aligned}
& \forall_{\alpha \in \mathscr{A}} \forall_{U, W \in 2^{X}}\left\{D_{1 ; 2^{X} ; \alpha}^{L-\mathscr{I}_{\mathrm{C} ; \mathcal{A}}}(U, W)\right. \\
& \left.=\max \left\{\sup _{u \in U} J_{\alpha}(u, W), \sup _{w \in W} J_{\alpha}(U, w)\right\}\right\}, \\
& \forall_{\alpha \in \mathscr{A}} \forall_{U, W \in 2^{X}}\left\{D_{2 ; 2^{X} ; \alpha}^{L-\mathscr{J}_{\mathrm{C} ; \mathcal{A}}}(U, W)\right. \\
& \left.=\max \left\{\sup _{u \in U} J_{\alpha}(u, W), \sup _{w \in W} J_{\alpha}(w, U)\right\}\right\}, \\
& \forall_{\alpha \in \mathscr{A}} \forall_{U, W \in 2^{X}}\left\{D_{3 ; 2^{X} ; \alpha}^{L-\mathscr{F}_{\mathrm{C} ; \mathscr{A}}}(U, W)=\sup _{u \in U} J_{\alpha}(u, W)\right\},
\end{aligned}
$$

then a family $\mathscr{D}_{\eta ; 2^{X}}^{L-\mathscr{F}_{C ; \mathscr{d}}}=\left\{D_{\eta ; 2^{X} ; \alpha}^{L-\mathscr{F}_{C} \mathscr{A}}, \alpha \in \mathscr{A}\right\}$ is said to be left $\mathscr{D}_{\eta ; 2^{X}}^{L-\mathscr{I}_{C ; A}}$-quasi-distance on $2^{X}$.

If

$$
\begin{aligned}
& \forall_{\alpha \in \mathscr{A}} \forall_{x, y \in X}\left\{C_{\alpha} \cdot D_{\eta ; 2^{X} ; \alpha}^{L-\mathcal{F}_{C ; \alpha}}(T(x), T(y))\right. \\
& \left.\quad \leq \lambda_{\alpha} J_{\alpha}(x, y)\right\},
\end{aligned}
$$

then we say that $(X, T)$ is a left $\left(\mathscr{D}_{\eta ; 2^{X}}^{L-\mathscr{I}_{C} ; \mathcal{A}}, \lambda\right)$-quasi-contraction on $X$.

(B) Let $\mathscr{J}_{C ; \mathscr{A}} \in \mathbb{J}_{\left(X, \mathscr{P}_{C ; \mathscr{A}}\right)}^{R}$. If

$$
\begin{aligned}
& \forall_{\alpha \in \mathscr{A}} \forall_{U, W \in 2^{X}}\left\{D_{1 ; 2^{X} ; \alpha}^{R-\mathscr{F}_{C ; \alpha}}(U, W)\right. \\
& \left.=\max \left\{\sup _{u \in U} J_{\alpha}(u, W), \sup _{w \in W} J_{\alpha}(U, w)\right\}\right\}, \\
& \forall_{\alpha \in \mathscr{A}} \forall_{U, W \in 2^{X}}\left\{D_{2 ; 2^{X} ; \alpha}^{R-\mathscr{F}_{C ; \mathcal{A}}}(U, W)\right. \\
& \left.=\max \left\{\sup _{u \in U} J_{\alpha}(u, W), \sup _{w \in W} J_{\alpha}(w, U)\right\}\right\}, \\
& \forall_{\alpha \in \mathscr{A}} \forall_{U, W \in 2^{X}}\left\{D_{3 ; 2^{X} ; \alpha}^{R-\mathscr{F}_{C ; \mathcal{A}}}(U, W)=\sup _{u \in U} J_{\alpha}(u, W)\right\},
\end{aligned}
$$

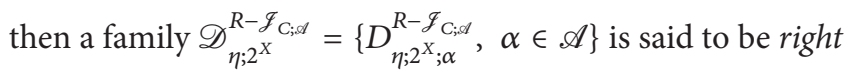
$\mathscr{D}_{\eta ; 2^{X}}^{R-\mathscr{I}_{C ; A}}$-quasi-distance on $2^{X}$.

If

$$
\begin{aligned}
& \forall_{\alpha \in \mathscr{A}} \forall_{x, y \in X}\left\{C_{\alpha} \cdot D_{\eta ; 2^{X} ; \alpha}^{R-\mathscr{I}_{C} ; \mathcal{A}}(T(x), T(y))\right. \\
& \left.\quad \leq \lambda_{\alpha} J_{\alpha}(x, y)\right\}
\end{aligned}
$$

then we say that $(X, T)$ is a right $\left(\mathscr{D}_{\eta ; 2^{X}}^{R-\mathscr{F}_{C ; A}}, \lambda\right)$-quasi-contraction on $X$.
Remark 24. Observe that $\mathscr{D}_{\eta ; 2^{X}}^{L-\mathscr{F}_{C ; A d}}$ and $\mathscr{D}_{\eta ; 2^{X}}^{R-\mathscr{F}_{C ; A}}$ extend (2). Quasi-contractions (23) and (25) extend (3).

Remark 25. Each $\left(\mathscr{D}_{\eta ; 2^{X}}^{L-\mathscr{J}_{C} ; \mathscr{A}}, \lambda\right)$-quasi-contraction $\left(\left(\mathscr{D}_{\eta ; 2^{X}}^{R-\mathscr{I}_{C} ; \mathscr{A}}\right.\right.$, $\lambda)$-quasi-contraction), $\eta \in\{1,2\}$, is $\left(\mathscr{D}_{3 ; 2^{X}}^{L-\mathscr{F}_{\mathrm{C} ; \mathcal{A}}}, \lambda\right)$-quasi-contraction $\left(\left(\mathscr{D}_{3 ; 2^{X}}^{R-\mathscr{F}_{\mathrm{C} ; A}}, \lambda\right)\right.$-quasi-contraction) but the converse does not necessarily hold.

\section{Convergence, Existence, Approximation, and Periodic Point Theorem of Nadler Type for Left (Right) Set-Valued Quasi-Contractions}

The following result extends Theorem 6 to spaces $\left(X, \mathscr{P}_{C ; \mathscr{A}}\right)$.

Theorem 26. Let $\left(X, \mathscr{P}_{C ; \mathscr{A}}\right)$ be the quasi-triangular space, and let $(X, T)$ be the set-valued dynamic system, $T: X \rightarrow 2^{X}$. Let $\eta \in\{1,2,3\}$, and let $\lambda=\left\{\lambda_{\alpha}\right\}_{\alpha \in \mathscr{A}} \in[0 ; 1)^{\mathscr{A}}$.

Assume that there exist a left (right) family $\mathscr{J}_{C ; A}$ generated by $\mathscr{P}_{C ; A}$ and a point $w^{0} \in X$ with the following properties.

(A1) $(X, T)$ is left $\left(\mathscr{D}_{\eta ; 2^{X}}^{L-\mathscr{F}_{C ; A}}, \lambda\right)$-quasi-contraction (right $\left(\mathscr{D}_{\eta ; 2^{X}}^{R-\mathscr{F}_{\mathrm{C} ; \mathcal{A}}}, \lambda\right)$-quasi-contraction) on $X$.

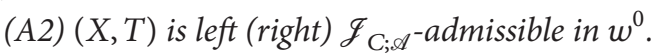

(A3) For every $x \in X$ and for every $\beta=\left\{\beta_{\alpha}\right\}_{\alpha \in \mathscr{A}} \in(0 ; \infty)^{\mathscr{A}}$ there exists $y \in T(x)$ such that

$$
\begin{gathered}
\forall_{\alpha \in \mathscr{A}}\left\{J_{\alpha}(x, y)<J_{\alpha}(x, T(x))+\beta_{\alpha}\right\}, \\
\left(\forall_{\alpha \in \mathscr{A}}\left\{J_{\alpha}(y, x)<J_{\alpha}(T(x), x)+\beta_{\alpha}\right\}\right) .
\end{gathered}
$$

Then the following hold.

(B1) There exist a dynamic process $\left(w^{m}: m \in\{0\} \cup \mathbb{N}\right)$ of the system $(X, T)$ starting at $w^{0}, \forall_{m \in\{0\} \cup \mathbb{N}}\left\{w^{m+1} \in T\left(w^{m}\right)\right\}$, and a point $w \in X$ such that $\left(w^{m}: m \in\{0\} \cup \mathbb{N}\right)$ is left (right) $\mathscr{P}_{C ; A}$-convergent to $w$.

(B2) If the set-valued dynamic system $\left(X, T^{[k]}\right)$ is left (right) $\mathscr{P}_{C ; \mathscr{A}}$-closed on $X$ for some $k \in \mathbb{N}$, then $\operatorname{Fix}\left(T^{[k]}\right) \neq \varnothing$ and there exist a dynamic process $\left(w^{m}: m \in\{0\} \cup \mathbb{N}\right)$ of the system $(X, T)$ starting at $w^{0}, \forall_{m \in\{0\} \cup \mathbb{N}}\left\{w^{m+1} \in T\left(w^{m}\right)\right\}$, and a point $w \in \operatorname{Fix}\left(T^{[k]}\right)$ such that $\left(w^{m}: m \in\{0\} \cup \mathbb{N}\right)$ is left (right)

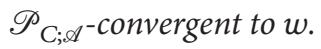

Proof. We prove only the case when $\mathscr{J}_{C ; \mathscr{A}}$ is a left family

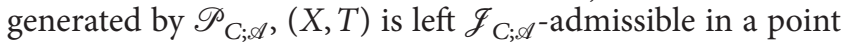
$w^{0} \in X$, and $\left(X, T^{[k]}\right)$ is left $\mathscr{P}_{C ; \mathscr{A}^{-}}$-losed on $X$. The case of "right" will be omitted, since the reasoning is based on the analogous technique.

Part 1. Assume that (A1)-(A3) hold.

By (21) and the fact that $J_{\alpha}: X^{2} \rightarrow[0 ; \infty), \alpha \in \mathscr{A}$, we choose

$$
r=\left\{r_{\alpha}\right\}_{\alpha \in \mathscr{A}} \in(0 ; \infty)^{\mathscr{A}}
$$


such that

$$
\forall_{\alpha \in \mathscr{A}}\left\{J_{\alpha}\left(w^{0}, T\left(w^{0}\right)\right)<\left(1-\frac{\lambda_{\alpha}}{C_{\alpha}}\right) r_{\alpha}\right\} .
$$

Put

$$
\forall_{\alpha \in \mathscr{A}}\left\{\beta_{\alpha}^{(0)}=\left(1-\frac{\lambda_{\alpha}}{C_{\alpha}}\right) r_{\alpha}-J_{\alpha}\left(w^{0}, T\left(w^{0}\right)\right)\right\} .
$$

In view of (28) and (29) this implies $\beta^{(0)}=\left\{\beta_{\alpha}^{(0)}\right\}_{\alpha \in \mathscr{A}} \epsilon$ $(0 ; \infty)^{\mathscr{A}}$ and we apply (26) to find $w^{1} \in T\left(w^{0}\right)$ such that

$$
\forall_{\alpha \in \mathscr{A}}\left\{J_{\alpha}\left(w^{0}, w^{1}\right)<J_{\alpha}\left(w^{0}, T\left(w^{0}\right)\right)+\beta_{\alpha}^{(0)}\right\} .
$$

We see from (30) and (31) that

$$
\forall_{\alpha \in \mathscr{A}}\left\{J_{\alpha}\left(w^{0}, w^{1}\right)<\left(1-\frac{\lambda_{\alpha}}{C_{\alpha}}\right) r_{\alpha}\right\} .
$$

Put now

$$
\forall_{\alpha \in \mathscr{A}}\left\{\beta_{\alpha}^{(1)}=\left(\frac{\lambda_{\alpha}}{C_{\alpha}}\right)\left[\left(1-\frac{\lambda_{\alpha}}{C_{\alpha}}\right) r_{\alpha}-J_{\alpha}\left(w^{0}, w^{1}\right)\right]\right\} .
$$

Then, in view of (32), we get $\beta^{(1)}=\left\{\beta_{\alpha}^{(1)}\right\}_{\alpha \in \mathscr{A}} \in(0 ; \infty)^{\mathscr{A}}$ and applying again (26) we find $w^{2} \in T\left(w^{1}\right)$ such that

$$
\forall_{\alpha \in \mathscr{A}}\left\{J_{\alpha}\left(w^{1}, w^{2}\right)<J_{\alpha}\left(w^{1}, T\left(w^{1}\right)\right)+\beta_{\alpha}^{(1)}\right\} .
$$

Observe that

$$
\forall_{\alpha \in \mathscr{A}}\left\{J_{\alpha}\left(w^{1}, w^{2}\right)<\left(\frac{\lambda_{\alpha}}{C_{\alpha}}\right)\left(1-\frac{\lambda_{\alpha}}{C_{\alpha}}\right) r_{\alpha}\right\} .
$$

Indeed, from (34), Definition 23(A), and using (33), in the event that $\eta=1$ or $\eta=2$ or $\eta=3$, we get

$$
\begin{aligned}
\forall_{\alpha \in \mathscr{A}} & \left\{J_{\alpha}\left(w^{1}, w^{2}\right)<J_{\alpha}\left(w^{1}, T\left(w^{1}\right)\right)+\beta_{\alpha}^{(1)}\right. \\
& \leq \sup \left\{J_{\alpha}\left(u, T\left(w^{1}\right)\right): u \in T\left(w^{0}\right)\right\}+\beta_{\alpha}^{(1)} \\
& \leq D_{\eta ; 2^{X} ; \alpha}^{L-\mathcal{F}_{i ;}}\left(T\left(w^{0}\right), T\left(w^{1}\right)\right)+\beta_{\alpha}^{(1)} \\
& \leq\left(\frac{\lambda_{\alpha}}{C_{\alpha}}\right) J_{\alpha}\left(w^{0}, w^{1}\right)+\beta_{\alpha}^{(1)} \\
& \left.=\left(\frac{\lambda_{\alpha}}{C_{\alpha}}\right)\left(1-\frac{\lambda_{\alpha}}{C_{\alpha}}\right) r_{\alpha}\right\} .
\end{aligned}
$$

Thus (35) holds.

Next define

$$
\begin{aligned}
& \forall_{\alpha \in \mathscr{A}}\left\{\beta_{\alpha}^{(2)}\right. \\
&\left.\quad=\left(\frac{\lambda_{\alpha}}{C_{\alpha}}\right)\left[\left(\frac{\lambda_{\alpha}}{C_{\alpha}}\right)\left(1-\frac{\lambda_{\alpha}}{C_{\alpha}}\right) r_{\alpha}-J_{\alpha}\left(w^{1}, w^{2}\right)\right]\right\} .
\end{aligned}
$$

Then, in view of (35), $\beta^{(2)}=\left\{\beta_{\alpha}^{(2)}\right\}_{\alpha \in \mathscr{A}} \in(0 ; \infty)^{\mathscr{A}}$. Applying (26) in this situation, we conclude that there exists $w^{3} \in$ $T\left(w^{2}\right)$ such that

$$
\forall_{\alpha \in \mathscr{A}}\left\{J_{\alpha}\left(w^{2}, w^{3}\right)<J_{\alpha}\left(w^{2}, T\left(w^{2}\right)\right)+\beta_{\alpha}^{(2)}\right\} .
$$

We seek to show that

$$
\forall_{\alpha \in \mathscr{A}}\left\{J_{\alpha}\left(w^{2}, w^{3}\right)<\left(\frac{\lambda_{\alpha}}{C_{\alpha}}\right)^{2}\left(1-\frac{\lambda_{\alpha}}{C_{\alpha}}\right) r_{\alpha}\right\}
$$

By (38), Definition 23(A), and using (37), in the event that $\eta=1$ or $\eta=2$ or $\eta=3$, it follows that

$$
\begin{aligned}
& \forall_{\alpha \in \mathscr{A}}\left\{J_{\alpha}\left(w^{2}, w^{3}\right)<J_{\alpha}\left(w^{2}, T\left(w^{2}\right)\right)+\beta_{\alpha}^{(2)}\right. \\
& \leqslant \sup _{u \in T\left(w^{1}\right)} J_{\alpha}\left(u, T\left(w^{2}\right)\right)+\beta_{\alpha}^{(2)} \\
& \leq D_{\eta ; 2^{X} ; \alpha}^{L-\mathscr{F}_{C} / \mathcal{A}}\left(T\left(w^{1}\right), T\left(w^{2}\right)\right)+\beta_{\alpha}^{(2)} \\
& \leq\left(\frac{\lambda_{\alpha}}{C_{\alpha}}\right) J_{\alpha}\left(w^{1}, w^{2}\right)+\beta_{\alpha}^{(2)} \\
& \left.=\left(\frac{\lambda_{\alpha}}{C_{\alpha}}\right)^{2}\left(1-\frac{\lambda_{\alpha}}{C_{\alpha}}\right) r_{\alpha}\right\} .
\end{aligned}
$$

Thus (39) holds.

Proceeding as before, using Definition 23(A), we get that there exists a sequence $\left(w^{m}: m \in \mathbb{N}\right)$ in $X$ satisfying

$$
\forall_{m \in \mathbb{N}}\left\{w^{m+1} \in T\left(w^{m}\right)\right\}
$$

and for calculational purposes, upon letting $\forall_{m \in \mathbb{N}}\left\{\beta^{(m)}=\right.$ $\left.\left\{\beta_{\alpha}^{(m)}\right\}_{\alpha \in \mathscr{A}}\right\}$ where

$$
\begin{aligned}
& \qquad \begin{aligned}
\forall_{\alpha \in \mathscr{A}} & \forall_{m \in \mathbb{N}}\left\{\beta_{\alpha}^{(m)}=\left(\frac{\lambda_{\alpha}}{C_{\alpha}}\right)\right. \\
\cdot & {\left.\left[\left(\frac{\lambda_{\alpha}}{C_{\alpha}}\right)^{m-1}\left(1-\frac{\lambda_{\alpha}}{C_{\alpha}}\right) r_{\alpha}-J_{\alpha}\left(w^{m-1}, w^{m}\right)\right]\right\} }
\end{aligned} \\
& \text { we observe that } \forall_{m \in \mathbb{N}}\left\{\beta^{(m)} \in(0 ; \infty)^{\mathscr{A}}\right\}, \\
& \forall_{\alpha \in \mathscr{A}} \forall_{m \in \mathbb{N}}\left\{J_{\alpha}\left(w^{m}, w^{m+1}\right)<J_{\alpha}\left(w^{m}, T\left(w^{m}\right)\right)\right. \\
& \left.+\beta_{\alpha}^{(m)}\right\}, \quad \\
& \forall_{\alpha \in \mathscr{A}} \forall_{m \in \mathbb{N}}\left\{J_{\alpha}\left(w^{m}, w^{m+1}\right)\right. \\
& \left.<\left(\frac{\lambda_{\alpha}}{C_{\alpha}}\right)^{m}\left(1-\frac{\lambda_{\alpha}}{C_{\alpha}}\right) r_{\alpha}\right\} .
\end{aligned}
$$


Let now $m<n$. Using $(\mathscr{J} 1)$, we get

$$
\begin{aligned}
& \forall_{\alpha \in \mathscr{A}}\left\{J_{\alpha}\left(w^{m}, w^{n}\right) \leqslant C_{\alpha} J_{\alpha}\left(w^{m}, w^{m+1}\right)\right. \\
& +C_{\alpha}^{2} J_{\alpha}\left(w^{m+1}, w^{m+2}\right)+\cdots \\
& +C_{\alpha}^{n-m-1} J_{\alpha}\left(w^{n-2}, w^{n-1}\right)+C_{\alpha}^{n-m-1} J_{\alpha}\left(w^{n-1}, w^{n}\right) \\
& =\sum_{j=0}^{n-m-2} C_{\alpha}^{j+1} J_{\alpha}\left(w^{m+j}, w^{m+j+1}\right) \\
& \left.+C_{\alpha}^{n-m-1} J_{\alpha}\left(w^{n-1}, w^{n}\right)\right\} .
\end{aligned}
$$

Hence, by (44), for each $\alpha \in \mathscr{A}$,

$$
\begin{aligned}
& J_{\alpha}\left(w^{m}, w^{n}\right)<\left(1-\frac{\lambda_{\alpha}}{C_{\alpha}}\right) \\
& \cdot r_{\alpha}\left[\sum_{j=0}^{n-m-2} C_{\alpha}^{j+1}\left(\frac{\lambda_{\alpha}}{C_{\alpha}}\right)^{m+j}+C_{\alpha}^{n-m-1}\left(\frac{\lambda_{\alpha}}{C_{\alpha}}\right)^{n-2}\right] \\
& =\left(1-\frac{\lambda_{\alpha}}{C_{\alpha}}\right) \\
& \cdot r_{\alpha}\left[C_{\alpha}\left(\frac{\lambda_{\alpha}}{C_{\alpha}}\right)^{m} \sum_{j=0}^{m-m-2} \lambda_{\alpha}^{j}+\left(\frac{C_{\alpha}}{\lambda_{\alpha}^{2}}\right) \frac{\lambda_{\alpha}^{n}}{C_{\alpha}^{m}}\right] .
\end{aligned}
$$

This and (41) mean that

$$
\exists_{\left(w^{m}: m \in \mathbb{N}\right)} \forall_{m \in\{0\} \cup \mathbb{N}}\left\{w^{m+1} \in T\left(w^{m}\right)\right\}
$$

and since $m<n$ implies $\lambda_{\alpha}^{n} \leq \lambda_{\alpha}^{m}$,

$$
\begin{aligned}
& \forall_{\alpha \in \mathscr{A}}\left\{\lim _{m \rightarrow \infty} \sup _{n>m} J_{\alpha}\left(w^{m}, w^{n}\right) \leq \lim _{m \rightarrow \infty} \sup _{n>m}\left(1-\frac{\lambda_{\alpha}}{C_{\alpha}}\right)\right. \\
& \cdot r_{\alpha}\left[C_{\alpha}\left(\frac{\lambda_{\alpha}}{C_{\alpha}}\right)^{m}\left(1-\lambda_{\alpha}\right)^{-1}+\left(\frac{C_{\alpha}}{\lambda_{\alpha}^{2}}\right) \frac{\lambda_{\alpha}^{n}}{C_{\alpha}^{m}}\right] \\
& \leq \lim _{m \rightarrow \infty}\left(1-\frac{\lambda_{\alpha}}{C_{\alpha}}\right) r_{\alpha}\left[C_{\alpha}\left(\frac{\lambda_{\alpha}}{C_{\alpha}}\right)^{m}\left(1-\lambda_{\alpha}\right)^{-1}\right. \\
& \left.\left.+\left(\frac{C_{\alpha}}{\lambda_{\alpha}^{2}}\right)\left(\frac{\lambda_{\alpha}}{C_{\alpha}}\right)^{m}\right]=0\right\} .
\end{aligned}
$$

Now, since $(X, T)$ is left $\mathscr{J}_{C ; \mathscr{A}}$-admissible in $w^{0} \in X$, by Definition 20(A), properties (47) and (48) imply that there exists $w \in X$ such that

$$
\forall_{\alpha \in \mathscr{A}}\left\{\lim _{m \rightarrow \infty} J_{\alpha}\left(w, w^{m}\right)=0\right\} .
$$

Next, defining $u_{m}=w^{m}$ and $w_{m}=w$ for $m \in \mathbb{N}$, by (48) and (49) we see that conditions (8) and (10) hold for the sequences $\left(u_{m}: m \in \mathbb{N}\right)$ and $\left(w_{m}: m \in \mathbb{N}\right)$ in $X$. Consequently, by $(\mathscr{J} 2)$, we get (12) which implies that

$$
\forall_{\alpha \in \mathscr{A}}\left\{\lim _{m \rightarrow \infty} p_{\alpha}\left(w, w^{m}\right)=\lim _{m \rightarrow \infty} p_{\alpha}\left(w_{m}, u_{m}\right)=0\right\}
$$

and so in particular we see that $w \in \operatorname{LIM}_{\left(w^{m}: m \in \mathbb{N}\right)}^{L-\mathscr{P}_{C ; A}}$.

Part 2. Assume that (A1)-(A3) hold and that, for some $k \in \mathbb{N}$,

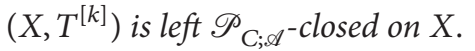

By Part 1, $\operatorname{LIM}_{\left(w^{m}: m \in\{0\} \cup \mathbb{N}\right)}^{L-\mathscr{P}_{C ; \mathscr{A}}} \neq \varnothing$ and since, by (47), $w^{(m+1) k} \in T^{[k]}\left(w^{m k}\right)$ for $m \in\{0\} \cup \mathbb{N}$, thus defining $\left(x_{m}=\right.$ $\left.w^{m-1+k}: m \in \mathbb{N}\right)$, we see that $\left(x_{m}: m \in \mathbb{N}\right) \subset T^{[k]}(X)$, $\operatorname{LIM}_{\left(x_{m}: m \in\{0\} \cup \mathbb{N}\right)}^{L-\mathscr{P}_{C ; \mathscr{A}}}=\operatorname{LIM}_{\left(w^{m}: m \in\{0\} \cup \mathbb{N}\right)}^{L-\mathscr{P}_{C ; A}} \neq \varnothing$, the sequences $\left(v_{m}=\right.$ $\left.w^{(m+1) k}: m \in \mathbb{N}\right) \subset T^{[k]}(X)$ and $\left(u_{m}=w^{m k}: m \in \mathbb{N}\right) \subset$ $T^{[k]}(X)$ satisfy $\forall_{m \in \mathbb{N}}\left\{v_{m} \in T^{[k]}\left(u_{m}\right)\right\}$ and, as subsequences of $\left(x_{m}: m \in\{0\} \cup \mathbb{N}\right)$, are left $\mathscr{P}_{C ; \mathscr{A}}$-converging to each point of the set $\operatorname{LIM}_{\left(w^{m}: m \in\{0\} \cup \mathbb{N}\right)}^{L-\mathscr{P}_{C ; \mathscr{A}}}$. Moreover, by Remark 18, $\operatorname{LIM}_{\left(w^{m}: m \in \mathbb{N}\right)}^{L-\mathscr{P}_{C ; \mathscr{d}}} \subset \operatorname{LIM}_{\left(v_{m}: m \in \mathbb{N}\right)}^{L-\mathscr{P}_{C ; \mathscr{d}}}$ and $\operatorname{LIM}_{\left(w^{m}: m \in \mathbb{N}\right)}^{L-\mathscr{P}_{C ; \mathscr{d}}} \subset \operatorname{LIM}_{\left(u_{m}: m \in \mathbb{N}\right)}^{L-\mathscr{P}_{C ;}}{ }^{L}$ By the above and by Definition 22, since $T^{[k]}$ is left $\mathscr{P}_{C ; \mathscr{A}^{-}}$ closed, we conclude that there exist $w \in \operatorname{LIM}_{\left(w^{m}: m \in\{0\} \cup \mathbb{N}\right)}^{L-\mathscr{P}_{C ; A}}=$ $\operatorname{LIM}_{\left(x_{m}: m \in \mathbb{N}\right)}^{L-\mathscr{P}_{C ; A}}$ such that $w \in T^{[k]}(w)$.

Part 3. The result now follows at once from Parts 1 and 2.

\section{Theorem of Banach Type in Quasi- Triangular Spaces $\left(X, \mathscr{P}_{C ; \mathscr{A}}\right)$}

In this section, in the quasi-triangular spaces $\left(X, \mathscr{P}_{C ; \mathscr{A}}\right)$, using left (right) families $\mathscr{J}_{C ; \mathscr{A}}$ generated by $\mathscr{P}_{C ; \mathscr{A}}$, we construct two types of left (right) single-valued quasicontractions $T: X \rightarrow X$, and convergence, existence, approximation, uniqueness, periodic point, and fixed point theorem for such quasi-contractions is also proved.

The following Definition 27 can be stated as a singlevalued version of Definition 23.

Definition 27. Let $\left(X, \mathscr{P}_{C ; \mathscr{A}}\right)$ be the quasi-triangular space, and let $\mathscr{J}_{C ; \mathscr{A}}$ be the left (right) family generated by $\mathscr{P}_{C ; \mathscr{A}}$. Let $(X, T)$ be the single-valued dynamic system, let $\lambda=$ $\left\{\lambda_{\alpha}\right\}_{\alpha \in \mathscr{A}} \in[0 ; 1)^{\mathscr{A}}$, and let $\eta \in\{1,2\}$.

(A) If $\mathscr{J}_{C ; \mathscr{A}} \in \mathbb{J}_{\left(X, \mathscr{P}_{C ; \mathscr{A}}\right)}^{L}$, then we define the left $\mathscr{D}_{X, \eta}^{L-\mathscr{F}_{C ; \mathscr{A}}}$ quasi-distance on $X$ by $\mathscr{D}_{X, \eta}^{L-\mathscr{F}_{\mathrm{C} ; \mathscr{A}}}=\left\{D_{\eta ; X ; \alpha}^{L-\mathscr{F}_{\mathrm{C} ; \mathscr{A}}}: X^{2} \rightarrow[0 ; \infty)\right.$, $\alpha \in \mathscr{A}\}$ where

$$
\begin{aligned}
& \forall_{\alpha \in \mathscr{A}} \forall_{u, w \in X}\left\{D_{1 ; X ; \alpha}^{L-\mathscr{J}_{C ; \mathscr{A}}}(u, w)\right. \\
& \left.=\max \left\{J_{\alpha}(u, w), J_{\alpha}(w, u)\right\}\right\}, \\
& \forall_{\alpha \in \mathscr{A}} \forall_{u, w \in X}\left\{D_{2 ; X ; \alpha}^{L-\mathscr{I}_{C ; \mathscr{d}}}(u, w)=J_{\alpha}(u, w)\right\} .
\end{aligned}
$$


One says that $(X, T)$ is left $\left(\mathscr{D}_{X, \eta}^{L-\mathcal{F}_{C ; / d}}, \lambda\right)$-quasi-contraction on $X$ if

$$
\begin{aligned}
& \forall_{\alpha \in \mathscr{A}} \forall_{x, y \in X}\left\{C_{\alpha} \cdot D_{\eta ; X ; \alpha}^{L-\mathcal{F}_{C ; A}}(T(x), T(y))\right. \\
& \left.\quad \leq \lambda_{\alpha} J_{\alpha}(x, y)\right\} .
\end{aligned}
$$

(B) If $\mathscr{J}_{C ; \mathscr{A}} \in \mathbb{J}_{\left(X, \mathscr{P}_{C ; d}\right)}^{R}$, then one defines the right $\mathscr{D}_{X, \eta}^{R-\mathscr{F}_{\mathrm{C} ; \alpha}}$-quasi-distance on $X$ by $\mathscr{D}_{X, \eta}^{R-\mathscr{F}_{\mathrm{C} ; \alpha}}=\left\{D_{\eta ; X ; \alpha}^{R-\mathscr{F}_{\mathrm{C} ; \alpha}}: X^{2} \rightarrow\right.$ $[0 ; \infty), \alpha \in \mathscr{A}\}$ where

$$
\begin{aligned}
& \forall_{\alpha \in \mathscr{A}} \forall_{u, w \in X}\left\{D_{1 ; \mathrm{X} ; \alpha}^{R-\mathscr{I}_{; ; \alpha}}(u, w)\right. \\
& \left.\quad=\max \left\{J_{\alpha}(u, w), J_{\alpha}(w, u)\right\}\right\}, \\
& \forall_{\alpha \in \mathscr{A}} \forall_{u, w \in X}\left\{D_{2 ; \mathrm{X} ; \alpha}^{R-\mathscr{F}_{\mathrm{C} ; \alpha}}(u, w)=J_{\alpha}(u, w)\right\} .
\end{aligned}
$$

One says that $(X, T)$ is right $\left(\mathscr{D}_{X, \eta}^{R-\mathscr{F}_{C ; \mathscr{q}}}, \lambda\right)$-quasi-contraction on $X$ if

$$
\begin{aligned}
& \forall_{\alpha \in \mathscr{A}} \forall_{x, y \in X}\left\{C_{\alpha} \cdot D_{\eta ; X ; \alpha}^{R-\mathscr{F}_{\mathrm{C} ; \boldsymbol{l}}}(T(x), T(y))\right. \\
& \left.\quad \leq \lambda_{\alpha} J_{\alpha}(x, y)\right\} .
\end{aligned}
$$

Remark 28. Observe that (52) and (54) extend (1). sequel.

The following terminologies will be much used in the

Definition 29. Let $\left(X, \mathscr{P}_{C ; \mathscr{A}}\right)$ be the quasi-triangular space, and let $\mathscr{F}_{C ; \mathscr{A} /}$ be the left (right) family generated by $\mathscr{P}_{C ; \mathscr{A} \text {. Let }}$ $(X, T)$ be the single-valued dynamic system, $T: X \rightarrow X$.

(A) Given $w^{0} \in X$, One says that $(X, T)$ is left (right) $\mathscr{J}_{\mathrm{C} ; \mathscr{A}}$-admissible in $w^{0}$ if, for the sequence $\left(w^{m}=T^{[m]}\left(w^{0}\right)\right.$ : $m \in\{0\} \cup \mathbb{N}), \operatorname{LIM}_{\left(w^{m}: m \in\{0\} \cup N\right)}^{L-\mathscr{F}_{C ;}} \neq \varnothing\left(\operatorname{LIM}_{\left(w^{m}: m \in\{0\} \cup N\right)}^{R-\mathscr{F}_{C ;}} \neq \varnothing\right)$ whenever

$$
\begin{gathered}
\forall_{\alpha \in \mathscr{A}}\left\{\lim _{m \rightarrow \infty} \sup _{n>m} J_{\alpha}\left(w^{m}, w^{n}\right)=0\right\} \\
\left(\forall_{\alpha \in \mathscr{A}}\left\{\lim _{m \rightarrow \infty} \sup _{n>m} J_{\alpha}\left(w^{n}, w^{m}\right)=0\right\}\right) .
\end{gathered}
$$

(B) We say that $(X, T)$ is left (right) $\mathscr{F}_{C ; \not A d}$-admissible on $X$, if $(X, T)$ is left (right) $\mathscr{J}_{C ; \mathscr{A}^{-}}$-admissible in each point $w^{0} \in X$.

Remark 30. Let $\left(X, \mathscr{P}_{C ; \mathscr{A}}\right)$ be the quasi-triangular space, and let $\mathscr{J}_{C ; \mathscr{A} A}$ be the left (right) family generated by $\mathscr{P}_{C ; \mathscr{A} A}$. Let $(X, T)$ be the single-valued dynamic system on $X$. If

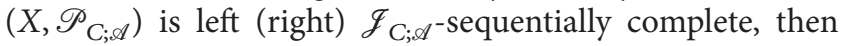
$(X, T)$ is left (right) $\mathscr{J}_{C ; \mathscr{A l}}$-admissible on $X$.

We can define the following generalization of continuity.

Definition 31. Let $\left(X, \mathscr{P}_{C ; \mathscr{A}}\right)$ be the quasi-triangular space. Let $(X, T)$ be the single-valued dynamic system, $T: X \rightarrow X$, and let $k \in \mathbb{N}$. The single-valued dynamic system $\left(X, T^{[k]}\right)$ is

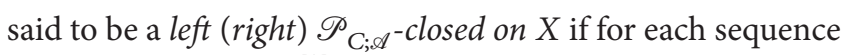

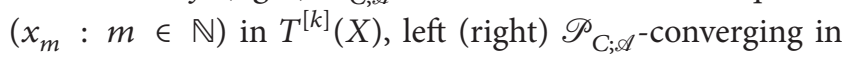
$X$ (thus $\left.\operatorname{LIM}_{\left(x_{m}: m \in \mathbb{N}\right)}^{L-\mathscr{P}_{C: q}} \neq \varnothing\left(\operatorname{LIM}_{\left(x_{m}: m \in \mathbb{N}\right)}^{R-\mathscr{P}_{G, \mathscr{A}}} \neq \varnothing\right)\right)$ and having subsequences $\left(v_{m}: m \in \mathbb{N}\right)$ and $\left(u_{m}: m \in \mathbb{N}\right)$ satisfying $\forall_{m \in \mathbb{N}}\left\{v_{m}=T^{[k]}\left(u_{m}\right)\right\}$; the following property holds: there exists $x \in \operatorname{LIM}_{\left(x_{m}: m \in \mathbb{N}\right)}^{L-\mathscr{P}_{G: d}}\left(x \in \operatorname{LIM}_{\left(x_{m}: m \in \mathbb{N}\right)}^{R-\mathscr{P}_{C ;}}\right)$ such that $x=$ $T^{[k]}(x)\left(x=T^{[k]}(x)\right)$.

The following result extends Theorem 5 to spaces ( $X$, $\mathscr{P}_{\text {C; } \mathbb{A}) \text {. }}$

Theorem 32. Let $\left(X, \mathscr{P}_{C ; \mathscr{A}}\right)$ be the quasi-triangular space, and let $(X, T)$ be the single-valued dynamic system, $T: X \rightarrow 2^{X}$. Let $\eta \in\{1,2\}$, and let $\lambda=\left\{\lambda_{\alpha}\right\}_{\alpha \in \mathscr{A}} \in[0 ; 1)^{\mathscr{A}}$.

Assume that there exist a left (right) family $\mathscr{F}_{\mathrm{C} ; \mathcal{A}}$ generated by $\mathscr{P}_{C ; A d}$ and a point $w^{0} \in X$ with the following properties.

(A1) $(X, T)$ is left $\left(\mathscr{D}_{X, \eta}^{L-\mathcal{F}_{C ; A}}, \lambda\right)$-quasi-contraction (right

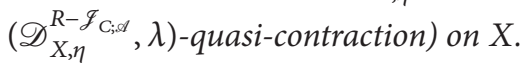

(A2) $(X, T)$ is left (right) $\mathscr{F}_{C ; \mathscr{A}}$-admissible in a point $w^{0} \in$ $X$.

Then the following hold.

(B1) There exists a point $w \in X$ such that the sequence $\left(w^{m}=T^{[m]}\left(w^{0}\right): m \in\{0\} \cup \mathbb{N}\right)$ starting at $w^{0}$ is left (right)

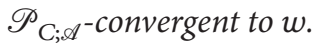

(B2) If the single-valued dynamic system $\left(X, T^{[k]}\right)$ is left

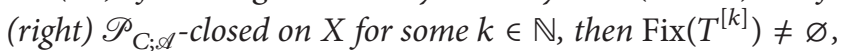
there exists a point $w \in \operatorname{Fix}\left(T^{[k]}\right)$ such that the sequence $\left(w^{m}=T^{[m]}\left(w^{0}\right): m \in\{0\} \cup \mathbb{N}\right)$ starting at $w^{0}$ is left (right)

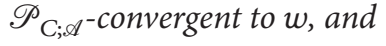

$$
\forall_{\alpha \in \mathscr{A}} \forall_{v \in \operatorname{Fix}\left(T^{[k]}\right)}\left\{J_{\alpha}(v, T(v))=J_{\alpha}(T(v), v)=0\right\} .
$$

(B3) If the family $\mathscr{P}_{C ; \mathscr{A}}=\left\{p_{\alpha}, \alpha \in \mathscr{A}\right\}$ is separating on $X$ and if the single-valued dynamic system $\left(X, T^{[k]}\right)$ is left (right) $\mathscr{P}_{C ; \mathscr{A}^{-}}$-closed on $X$ for some $k \in \mathbb{N}$, then there exists a point $w \in X$ such that

$$
\operatorname{Fix}\left(T^{[k]}\right)=\operatorname{Fix}(T)=\{w\},
$$

the sequence $\left(w^{m}=T^{[m]}\left(w^{0}\right): m \in\{0\} \cup \mathbb{N}\right)$ starting at $w^{0}$ is left (right) $\mathscr{P}_{C ; \mathbb{A}^{-}}$convergent to $w$, and

$$
\forall_{\alpha \in \mathscr{A}}\left\{J_{\alpha}(w, w)=0\right\} .
$$

Proof. By Theorem 26, we prove only (56)-(58) and only in the case of "left." We omit the proof in the case of "right," which is based on the analogous technique.

Part 1. Property (56) holds. Suppose that $\exists_{\alpha_{0} \in \mathscr{A}} \exists_{v \in \mathrm{Fix}\left(T^{[k]}\right)}\left\{J_{\alpha_{0}}(v\right.$, $T(v))>0\}$. Of course, $v=T^{[k]}(v)=T^{[2 k]}(v), T(v)=$ $T^{[2 k]}(T(v))$ and, for $\eta \in\{1,2\}$, by Definition 27(A),

$$
\begin{aligned}
0 & <J_{\alpha_{0}}(v, T(v))=J_{\alpha_{0}}\left(T^{[2 k]}(v), T^{[2 k]}(T(v))\right) \\
& \leq D_{\eta ; X ; \alpha_{0}}^{L-\mathscr{F}_{C ;}}\left(T^{[2 k]}(v), T^{[2 k]}(T(v))\right)
\end{aligned}
$$




$$
\begin{aligned}
& \leq\left(\frac{\lambda_{\alpha_{0}}}{C_{\alpha_{0}}}\right) J_{\alpha_{0}}\left(T^{[2 k-1]}(v), T^{[2 k-1]}(T(v))\right) \\
& \leq\left(\frac{\lambda_{\alpha_{0}}}{C_{\alpha_{0}}}\right) \cdot D_{\eta ; X ; \alpha_{0}}^{L-\mathcal{F}_{;}, \mathcal{A}}\left(T^{[2 k-1]}(v), T^{[2 k-1]}(T(v))\right) \\
& \leq\left(\frac{\lambda_{\alpha_{0}}}{C_{\alpha_{0}}}\right)^{2} J_{\alpha_{0}}\left(T^{[2 k-2]}(v), T^{[2 k-2]}(T(v))\right) \leq \cdots \\
& \leq\left(\frac{\lambda_{\alpha_{0}}}{C_{\alpha_{0}}}\right)^{2 k} J_{\alpha_{0}}(v, T(v))<J_{\alpha_{0}}(v, T(v)),
\end{aligned}
$$

which is impossible. Therefore,

$$
\forall_{\alpha \in \mathscr{A}} \forall_{v \in \operatorname{Fix}\left(T^{[k]}\right)}\left\{J_{\alpha}(v, T(v))=0\right\} .
$$

Suppose now that $\exists_{\alpha_{0} \in \mathscr{A}} \exists_{v \in \operatorname{Fix}\left(T^{[k]}\right)}\left\{J_{\alpha_{0}}(T(v), v)>0\right\}$. Then, by Definition 27(A) and property (60), using the fact that $v=$ $T^{[k]}(v)=T^{[2 k]}(v)$, we get, for $\eta \in\{1,2\}$, that

$$
\begin{aligned}
0< & J_{\alpha_{0}}(T(v), v)=J_{\alpha_{0}}\left(T^{[k+1]}(v), T^{[2 k]}(v)\right) \\
\leq & \sum_{m=1}^{k-2} C_{\alpha_{0}}^{m} J_{\alpha_{0}}\left(T^{[k+m]}(v), T^{[k+m+1]}(v)\right) \\
& +C_{\alpha_{0}}^{k-2} J_{\alpha_{0}}\left(T^{[2 k-1]}(v), T^{[2 k]}(v)\right) \\
\leq & \sum_{m=1}^{k-2} C_{\alpha_{0}}^{m} \cdot D_{\eta ; X ; \alpha_{0}}^{L-\mathscr{f}_{\mathrm{C} ; \boldsymbol{d}}}\left(T^{[k+m]}(v), T^{[k+m+1]}(v)\right) \\
& +C_{\alpha_{0}}^{k-2} \cdot D_{\eta ; X ; \alpha_{0}}^{L-\mathscr{F}_{C ;}}\left(T^{[2 k-1]}(v), T^{[2 k]}(v)\right) \\
\leq & \sum_{m=1}^{k-2} C_{\alpha_{0}}^{m}\left(\frac{\lambda_{\alpha_{0}}}{C_{\alpha_{0}}}\right)^{k+m} J_{\alpha_{0}}(v, T(v)) \\
& +C_{\alpha_{0}}^{k-2}\left(\frac{\lambda_{\alpha_{0}}}{C_{\alpha_{0}}}\right)^{2 k-1} J_{\alpha_{0}}(v, T(v))=0,
\end{aligned}
$$

which is impossible. Therefore,

$$
\forall_{\alpha \in \mathscr{A}} \forall_{v \in \operatorname{Fix}\left(T^{[k]}\right)}\left\{J_{\alpha}(T(v), v)=0\right\} .
$$

We see that (56) is a consequence of (60) and (62).

Part 2. Properties (57) and (58) hold. We first observe that

$$
\forall_{v \in \operatorname{Fix}\left(T^{[k]}\right)}\{T(v)=v\} ;
$$

in other words, $\operatorname{Fix}\left(T^{[k]}\right)=\operatorname{Fix}(T)$. In fact, if $v \in \operatorname{Fix}\left(T^{[k]}\right)$ and $T(v) \neq v$, then, since the family $\mathscr{P}_{C ; \mathscr{A}}=\left\{p_{\alpha}, \alpha \in \mathscr{A}\right\}$ is separating on $X$, we get that $T(v) \neq v \Rightarrow \exists_{\alpha \in \mathscr{A}}\left\{p_{\alpha}(T(v), v)>\right.$ $\left.0 \vee p_{\alpha}(v, T(v))>0\right\}$. In view of Theorem 15 this implies $T(v) \neq$ $v \Rightarrow \exists_{\alpha \in \mathscr{A}}\left\{J_{\alpha}(T(v), v)>0 \vee J_{\alpha}(v, T(v))>0\right\}$. However, by property (56), this is impossible.

Next we see that $\forall_{v \in \operatorname{Fix}\left(T^{[k]}\right)=\operatorname{Fix}(T)}\left\{J_{\alpha}(v, v)=0\right\}$. In fact, by Definition 11(A) and property (56), we conclude that $\forall_{\alpha \in \mathscr{A}} \forall_{v \in \operatorname{Fix}\left(T^{[k]}\right)}\left\{J_{\alpha}(v, v) \leq C_{\alpha}\left[J_{\alpha}(v, T(v))+J_{\alpha}(T(v), v)\right]=0\right\}$.
Finally, suppose that $u, w \in \operatorname{Fix}(T)$ and $u \neq w$. Then, since the family $\mathscr{P}_{C ; \mathscr{A}}=\left\{p_{\alpha}, \alpha \in \mathscr{A}\right\}$ is separating on $X$, we get $\exists_{\alpha_{0} \in \mathscr{A}}\left\{p_{\alpha_{0}}(u, w)>0 \vee p_{\alpha_{0}}(w, u)>0\right\}$. By applying Theorem 15, this implies $\exists_{\alpha_{0} \in \mathscr{A}}\left\{J_{\alpha_{0}}(u, w)>0 \vee J_{\alpha_{0}}(w, u)>\right.$ $0\}$. Consequently, for $\eta \in\{1,2\}$, by Definition 27(A), we conclude that

$$
\begin{aligned}
& \exists_{\alpha_{0} \in \mathscr{A}}\left\{\left[J_{\alpha_{0}}(u, w)>0, J_{\alpha_{0}}(u, w)\right.\right. \\
& \quad=J_{\alpha_{0}}(T(u), T(w)) \leq D_{\eta ; X ; \alpha_{0}}^{L-\mathcal{F}_{C} / \mathscr{A}}(T(u), T(w)) \\
& \left.\quad \leq\left(\frac{\lambda_{\alpha_{0}}}{C_{\alpha_{0}}}\right) J_{\alpha_{0}}(u, w)<J_{\alpha_{0}}(u, w)\right] \text { or }\left[J_{\alpha_{0}}(w, u)\right. \\
& \quad>0, J_{\alpha_{0}}(w, u)=J_{\alpha_{0}}(T(w), T(u)) \\
& \quad \leq D_{\eta ; X ; \alpha_{0}}^{L-\mathscr{F}_{C} ; \mathcal{A}}(T(w), T(u)) \leq\left(\frac{\lambda_{\alpha_{0}}}{C_{\alpha_{0}}}\right) J_{\alpha_{0}}(w, u) \\
& \left.\left.\quad<J_{\alpha_{0}}(w, u)\right]\right\}
\end{aligned}
$$

which is impossible. This gives that $\operatorname{Fix}(T)$ is a singleton.

Thus (57) and (58) hold.

\section{Examples of Spaces $\left(X, \mathscr{P}_{C ; \mathscr{A}}\right)$}

Example 1. Let $X=[0 ; 6], \gamma \geq 81$ and let $p: X^{2} \rightarrow[0 ; \infty)$ be of the form

$$
\begin{aligned}
& p(u, w) \\
& = \begin{cases}0 & \text { if } u \geq w, \quad\{u, w\} \cap(0 ; 6)=\{u, w\}, \\
(w-u)^{4} & \text { if } u<w,\{u, w\} \cap(0 ; 6)=\{u, w\}, \\
\gamma & \text { if }\{u, w\} \cap(0 ; 6) \neq\{u, w\} .\end{cases}
\end{aligned}
$$

(1) $\left(X, \mathscr{P}_{\{8\} ;\{1\}}\right), \mathscr{P}_{\{8\} ;\{1\}}=\{p\}$, is the quasi-triangular space. In fact,

$$
\forall_{u, v, w \in X}\{p(u, w) \leq 8[p(u, v)+p(v, w)]\} .
$$

Inequality (66) is a consequence of Cases 1-6.

Case 1. If $u, v, w \in(0 ; 6)$ and $v \leq u<w$, then $p(u, v)=0$ and $w-u \leq w-v$. This gives $p(u, w)=(w-u)^{4} \leq(w-v)^{4}<$ $8(w-v)^{4}=8[p(u, v)+p(v, w)]$.

Case 2. If $u, v, w \in(0 ; 6), u<w$ and $u \leq v \leq w$, then $p(u, w)=$ $(w-u)^{4}$ and $f\left(v_{0}\right)=\min _{u \leq v \leq w} f(v)=(w-u)^{4}$ where, for $u \leq v \leq w, f(v)=8[p(u, v)+p(v, w)]=8\left[(v-u)^{4}+(w-v)^{4}\right]$ and $v_{0}=(u+w) / 2$.

Case 3. $\sup _{u, w \in(0 ; 6) ; u<w} p(u, w)=\sup _{u, w \in(0 ; 6) ; u<w}(w-u)^{4}=$ $6^{4}=1296$ and $\sup _{u, w \in(0 ; 6) ; u<w} \min _{u \leq v \leq w} 8[p(u, v)+p(v, w)]=$ $\sup _{u, w \in(0 ; 6) ; u<w} \min _{u \leq v \leq w} 8\left[(v-u)^{4}+(w-v)^{4}\right]=8\left[(3-0)^{4}+\right.$ $\left.(6-3)^{4}\right]=8[81+81]=1296$. 
Case 4. If $u, v, w \in(0 ; 6)$ and $u<w \leq v$, then $p(v, w)=0$ and $w-u \leq v-u$. This gives $p(u, w)=(w-u)^{4} \leq(v-u)^{4}<$ $8(v-u)^{4}=8[p(u, v)+p(v, w)]$.

Case 5. If $u, w \in(0 ; 6), u<w$ and $v \in\{0,6\}$, then $p(u, w) \leq$ $1296 \leq 8[p(u, v)+p(v, w)]=8[\gamma+\gamma]$.

Case 6. If $\{u, w\} \cap(0 ; 6) \neq\{u, w\}$, then $\forall_{v \in X}\{p(u, w)=\gamma<$ $8 \gamma \leq 8[p(u, v)+p(v, w)]\}$.

(2) $\mathscr{P}_{\{8\} ;\{1\}}=\{p\}$ is asymmetric. Indeed, we have that $0=$ $p(5,1) \neq p(1,5)=256$. Therefore, condition $\forall_{u, w \in X}\{p(u$, $w)=p(w, u)\}$ does not hold.

(3) $\mathscr{P}_{\{8\} ;\{1\}}=\{p\}$ does not vanish on the diagonal. Indeed, if $u \in\{0,6\}$, then $p(u, u)=\gamma \neq 0$. Therefore, the condition $\forall_{u \in X}\{p(u, u)=0\}$ does not hold.

(4) For the constant sequence of the form $\left(u_{m}=3\right.$ : $m \in \mathbb{N}) \subset X$ the sets $\operatorname{LIM}_{\left(u_{m}: m \in \mathbb{N}\right)}^{L-\mathscr{P}_{\{8\} ; 1\}}}$ and $\operatorname{LIM}_{\left(u_{m}: m \in \mathbb{N}\right)}^{R-\mathscr{P}_{\{8\} ;\{1\}}}$ are not singletons. Indeed, by (65), Remark 12, and Definition 16(B), we have that $\operatorname{LIM}_{\left(u_{m}: m \in \mathbb{N}\right)}^{L-\mathscr{P}_{\{8\} ;\{1\}}}=[3 ; 6]$ and $\operatorname{LIM}_{\left(u_{m}: m \in \mathbb{N}\right)}^{R-\mathscr{P}_{\{8\} ;\{1\}}}=[0 ; 3]$.

Example 2. Let $X$ be a set (nonempty), $A \subset X, A \neq \varnothing, A \neq X$, $\gamma>0$, and let $p: X^{2} \rightarrow[0 ; \infty)$ be of the form

$$
p(u, w)= \begin{cases}0 & \text { if } A \cap\{u, w\}=\{u, w\} \\ \gamma & \text { if } A \cap\{u, w\} \neq\{u, w\} .\end{cases}
$$

(1) A pair $\left(X, \mathscr{P}_{\{1\} ;\{1\}}\right), \mathscr{P}_{\{1\} ;\{1\}}=\{p\}$, is the quasi-triangular space. Indeed, formula (67) yields $\forall_{u, v, w \in X}\{q(u, w) \leq$ $q(u, v)+q(v, w)\}$. Otherwise, $\exists_{u_{0}, v_{0}, w_{0} \in X}\left\{q\left(u_{0}, w_{0}\right)>q\left(u_{0}\right.\right.$, $\left.\left.v_{0}\right)+q\left(v_{0}, w_{0}\right)\right\}$. It is clear that then $q\left(u_{0}, w_{0}\right)=\gamma, q\left(u_{0}, v_{0}\right)=$ 0 , and $q\left(v_{0}, w_{0}\right)=0$. From this we see that $A \cap\left\{u_{0}, w_{0}\right\} \neq$ $\left\{u_{0}, w_{0}\right\}, A \cap\left\{u_{0}, v_{0}\right\}=\left\{u_{0}, v_{0}\right\}$, and $A \cap\left\{v_{0}, w_{0}\right\}=\left\{v_{0}, w_{0}\right\}$. This is impossible.

(2) $\mathscr{P}_{\{1\} ;\{1\}}=\{p\}$ does not vanish on the diagonal. Indeed, if $u \in X \backslash A$, then $p(u, u)=\gamma \neq 0$. Therefore, the condition $\forall_{u \in X}\{p(u, u)=0\}$ does not hold.

(3) $\mathscr{P}_{\{1\} ;\{1\}}=\{p\}$ is symmetric. This follows from (67).

(4) We observe that $\operatorname{LIM}_{\left(u_{m}: m \in \mathbb{N}\right)}^{L-\mathscr{P}_{\{1\} ; 1\}}}=\operatorname{LIM}_{\left(u_{m}: m \in \mathbb{N}\right)}^{R-\mathscr{P}_{\{1\} ; 1\}}}=$ A for each sequence $\left(u_{m}: m \in \mathbb{N}\right) \subset A$. We conclude this from (67).

Example 3. Let $X=[0 ; 6]$ and let $p: X^{2} \rightarrow[0 ; \infty)$ be of the form

$$
p(u, w)= \begin{cases}0 & \text { if } u \geq w \\ (w-u)^{3} & \text { if } u<w .\end{cases}
$$

(1) $\left(X, \mathscr{P}_{\{4\} ;\{1\}}\right), \mathscr{P}_{\{4\} ;\{1\}}=\{p\}$, is the quasi-triangular space. In fact, $\forall_{u, v, w \in X}\{q(u, w) \leq 4[q(u, v)+q(v, w)]\}$ holds. This is a consequence of Cases 1-3.

Case 1. If $v \leq u<w$, then $p(u, v)=0, w-u \leq w-v$, and, consequently, $p(u, w)=(w-u)^{3} \leq(w-v)^{3}<4(w-v)^{3}=$ $4 p(v, w)=4[p(u, v)+p(v, w)]$.

Case 2. If $u<w$ and $u \leq v \leq w$, then $q(u, w)=(w-u)^{3}$ and $f\left(v_{0}\right)=\min _{u \leqslant v \leqslant w} f(v)=(w-u)^{3}$ where $v_{0}=(u+w) / 2$ is a minimum point of the map $f(v)=4[p(u, v)+p(v, w)]=$ $4(w-u)\left[w^{2}+w u+u^{2}+3 v^{2}-3 v(w+u)\right]$.

Case 3. If $u<w \leq v$, then $p(v, w)=0$ and, consequently, $p(u, w)=(w-u)^{3} \leq(v-u)^{3}<4(v-u)^{3}=4 p(u, v)=4[p(u$, $v)+p(v, w)]$.

(2) $\mathscr{P}_{\{4\} ;\{1\}}=\{p\}$ is asymmetric. Indeed, we have that $0=$ $p(6,0) \neq p(0,6)=216$. Therefore, condition $\forall_{u, w \in X}\{p(u$, $w)=p(w, u)\}$ does not hold.

(3) $\mathscr{P}_{\{4\} ;\{1\}}=\{p\}$ vanishes on the diagonal. In fact, by (68), it is clear that $\forall_{u \in X}\{p(u, u)=0\}$.

(4) We observe that $\operatorname{LIM}_{\left(u_{m}: m \in \mathbb{N}\right)}^{L-\mathscr{P}_{\{4\} ; 1\}}}=[2 ; 6]$ and $\operatorname{LIM}_{\left(u_{m}: m \in \mathbb{N}\right)}^{R-\mathscr{P}_{\{4\} ; 1\}}}=[1 ; 2]$ for sequence $\left(u_{m}=2: m \in \mathbb{N}\right)$. We conclude this from (68).

Example 4. Let $X=[0 ; 6]$ and let $\mathscr{P}_{\{2\} ;\{1\}}=\{p\}$ where $p$ : $X^{2} \rightarrow[0 ; \infty)$ is of the form

$$
p(u, w)= \begin{cases}0 & \text { if } u \geq w \\ (u-w)^{2} & \text { if } u<w .\end{cases}
$$

Let

$$
E=[0 ; 3) \cup(3 ; 6]
$$

and let $\mu \geq 36 / 4$ and $\mathscr{J}_{\{2\} ;\{1\}}=\{J\}$ where $J: X^{2} \rightarrow[0 ; \infty)$ is of the form

$$
J(u, w)= \begin{cases}p(u, w) & \text { if } E \cap\{u, w\}=\{u, w\}, \\ \mu & \text { if } E \cap\{u, w\} \neq\{u, w\} .\end{cases}
$$

(1) $\mathscr{J}_{\{2\} ;\{1\}}$ is not symmetric. In fact, by $(69)-(71), J(0,6)=$ 36 and $J(6,0)=0$.

(2) $\mathscr{J}_{\{2\} ;\{1\}}=\{J\} \in \mathbb{J}_{\left(X, \mathscr{P}_{\{2\} ;\{1\}}\right)}^{L} \cap \mathbb{J}_{\left(X, \mathscr{P}_{\{2\} ; 1\}}\right)}^{R}$. See Theorem 14.

Remark 33. By Examples 1-4 it follows that the distances $p$ defined by (65) and (67)-(69) and $J$ defined by (70) and (71) are not metrics, ultra metrics, quasi-metrics, ultra quasimetrics, $b$-metrics, partial metrics, partial $b$-metrics, pseudometrics (gauges), quasi-pseudometrics (quasi-gauges), and ultra quasi-pseudometrics (ultra quasi-gauges).

\section{Examples Illustrating Theorem 26}

Example 1. Let $X=[0 ; 6]$, let $\gamma>2048$ be arbitrary and fixed, and, for $u, w \in X$, let

$$
\begin{aligned}
& p(u, w) \\
& = \begin{cases}0 & \text { if } u \geq w,\{u, w\} \cap(0 ; 6)=\{u, w\}, \\
(w-u)^{4} & \text { if } u<w,\{u, w\} \cap(0 ; 6)=\{u, w\}, \\
\gamma & \text { if }\{u, w\} \cap(0 ; 6) \neq\{u, w\} .\end{cases}
\end{aligned}
$$

Define the set-valued dynamic system $(X, T)$ by

$$
T(u)= \begin{cases}{[1 ; 2]} & \text { if } u \in[0 ; 3) \cup(4 ; 6] \\ (4 ; 6) & \text { if } u \in[3 ; 4]\end{cases}
$$


Let

$$
E=[0 ; 3) \cup(4 ; 6]
$$

and let $J: X \times X \rightarrow[0 ; \infty)$ be of the form

$$
J(u, w)= \begin{cases}p(u, w) & \text { if } E \cap\{u, w\}=\{u, w\}, \\ \gamma & \text { if } E \cap\{u, w\} \neq\{u, w\} .\end{cases}
$$

(1) $\left(X, \mathscr{P}_{\{8\} ;\{1\}}\right)$, where $\mathscr{P}_{\{8\} ;\{1\}}=\{p\}$, is the quasitriangular space, and $\mathscr{J}_{\{8\} ;\{1\}}=\{J\}$ is the left and right family generated by $\mathscr{P}_{\{8\} ;\{1\}}$. This is a consequence of Definitions 7 and 11, Example 1, and Theorem 14; we see that $\gamma=\mu>81$.

(2) $(X, T)$ is a $\left(D=\mathscr{D}_{1 ; 2^{X}}^{L-\mathcal{F}_{\{8 ;: 11\}}}=\mathscr{D}_{1 ; 2^{X}}^{R-\mathcal{L}_{\{8 ; ; 1\}}}, \lambda \in[2048 /\right.$ $\gamma ; 1))$-quasi-contraction on $X$; that is, $\forall_{\lambda \in[2048 / \gamma ; 1)} \forall_{x, y \in X}\{8$. $D(T(x), T(y)) \leq \lambda J(x, y)\}$ where

$$
\begin{array}{r}
D(U, W)=\max \left\{\sup _{u \in U} J(u, W), \sup _{w \in W} J(U, w)\right\}, \\
U, W \in 2^{X} .
\end{array}
$$

Indeed, we see that this follows from (73)-(76) and from Cases 1-4 below.

Case 1. If $x, y \in[0 ; 3) \cup(4 ; 6]$, then $T(x)=T(y)=[1 ; 2]=U \subset$ $E$ and $\sup _{u \in U}\left\{\inf _{w \in U} J(u, w)\right\}=\sup _{u \in U}\{J(u, u)=p(u, u)=$ $0\}=0$. Thus $4 D(T(x), T(y))=0 \leq \lambda J(x, y)$.

Case 2. If $x \in[0 ; 3) \cup(4 ; 6]$ and $y \in[3 ; 4]$, then $T(x)=[1 ; 2]=$ $U \subset E, T(y)=(4 ; 6)=W \subset E$, and $\sup _{u \in U}\left\{\inf _{w \in W} J(u, w)\right\}=$ $\sup _{u \in U}\left\{\inf _{w \in W}(w-u)^{4}\right\}=\sup _{u \in U}(4-u)^{4}=81$ and $\sup _{w \in W}\left\{\inf _{u \in U} J(u, w)\right\}=\sup _{w \in W}\left\{\inf _{u \in U}(w-u)^{4}\right\}=$ $\sup _{w \in W}(w-2)^{4}=256$. Thus $8 D(T(x), T(y))=2048$. On the other hand, $y \notin E$ which gives $J(x, y)=\gamma$. Therefore, $8 D(T(x), T(y)) \leq \lambda J(x, y)$ whenever $2048 \leq \lambda \gamma$. This gives $2048 / \gamma \leq \lambda<1$ whenever $\gamma>\max \{2048 ; 81\}$.

Case 3. If $x \in[3 ; 4]$ and $y \in[0 ; 3) \cup(4 ; 6]$, then $T(x)=$ $(4 ; 6)=U \subset E$ and $T(y)=[1 ; 2]=W \subset E$. Hence we obtain $\sup _{u \in \mathrm{U}}\left\{\inf _{w \in W} J(u, w)\right\}=\sup _{u \in U}\left\{\inf _{w \in W} p(u, w)\right\}=$ $\sup _{w \in W}\left\{\inf _{u \in U} J(u, w)\right\}=\sup _{w \in W}\left\{\inf _{u \in U} p(u, w)\right\}=0$. Therefore, $8 D(T(x), T(y))=0 \leq \lambda J(x, y)$.

Case 4. If $x, y \in[3 ; 4]$, then $T(x)=T(y)=(4 ; 6)=U \subset E$. Therefore $4 D_{1}(T(x), T(y))=0 \leq \lambda J(x, y)$.

(3) Property (26) holds; that is, $\forall_{x \in X} \forall_{\beta \in(0 ; \infty)} \exists_{y \in T(x)}\{J(x$, $y)<J(x, T(x))+\beta\}$. Indeed, this follows from Cases 1-4 below.

Case 1. If $x_{0}=0$ and $y_{0}=1 \in T\left(x_{0}\right)=[1 ; 2]$, then $J\left(x_{0}, y_{0}\right)=$ $\gamma, J\left(x_{0}, T\left(x_{0}\right)\right)=\inf _{w \in[1 ; 2]} J\left(x_{0}, w\right)=\gamma$, and $\forall_{\beta \in(0 ; \infty)}\left\{J\left(x_{0}\right.\right.$, $\left.\left.y_{0}\right)<J\left(x_{0}, T\left(x_{0}\right)\right)+\beta\right\}$.

Case 2. If $x_{0} \in(0 ; 1]$ and $y_{0}=1 \in T\left(x_{0}\right)=[1 ; 2]$, then $J\left(x_{0}, y_{0}\right)=1-x_{0}, J\left(x_{0}, T\left(x_{0}\right)\right)=\inf _{w \in[1 ; 2]} J\left(x_{0}, w\right)=1-x_{0}$, and $\forall_{\beta \in(0 ; \infty)}\left\{J\left(x_{0}, y_{0}\right)<J\left(x_{0}, T\left(x_{0}\right)\right)+\beta\right\}$.

Case 3. If $x_{0} \in(1 ; 3) \cup(4 ; 6)$ and $y_{0}=1 \in T\left(x_{0}\right)=[1 ; 2]$, then $J\left(x_{0}, y_{0}\right)=0, J\left(x_{0}, T\left(x_{0}\right)\right)=0$, and $\forall_{\beta \in(0 ; \infty)}\left\{J\left(x_{0}, y_{0}\right)<\right.$ $\left.J\left(x_{0}, T\left(x_{0}\right)\right)+\beta\right\}$.
Case 4. If $x_{0} \in[3 ; 4]$ and $y_{0} \in T\left(x_{0}\right)=(4 ; 6)$, then $J\left(x_{0}, y_{0}\right)=$ $\gamma, p\left(x_{0}, T\left(x_{0}\right)\right)=\gamma$, and $\forall_{\beta \in(0 ; \infty)}\left\{J\left(x_{0}, y_{0}\right)<J\left(x_{0}, T\left(x_{0}\right)\right)+\right.$ $\beta\}$.

Case 5. If $x_{0}=6$ and $y_{0} \in T\left(x_{0}\right)=[1 ; 2]$, then $J\left(x_{0}, y_{0}\right)=\gamma$, $p\left(x_{0}, T\left(x_{0}\right)\right)=\gamma$, and $\forall_{\beta \in(0 ; \infty)}\left\{J\left(x_{0}, y_{0}\right)<J\left(x_{0}, T\left(x_{0}\right)\right)+\beta\right\}$.

(4) $(X, T)$ is left and right $\mathscr{F}_{\{8\} ;\{1\}}$-admissible in each point $w^{0} \in X$. In fact, if $w^{0} \in X$ and $\left(w^{m}: m \in\{0\} \cup \mathbb{N}\right)$ are such that $\forall_{m \in\{0\} \cup \mathbb{N}}\left\{w^{m+1} \in T\left(w^{m}\right)\right\}$ and $\lim _{m \rightarrow \infty} \sup _{n>m} J\left(w^{m}, w^{n}\right)=$ $0\left(\lim _{m \rightarrow \infty} \sup _{n>m} J\left(w^{n}, w^{m}\right)=0\right)$, then $\forall_{m \geq 2}\left\{w^{m} \in[1 ; 2]\right\}$ and, consequently, by (72), $\forall_{w \in[2 ; 6) \subset X}\left\{\lim _{m \rightarrow \infty} p\left(w, w^{m}\right)=\right.$ 0\} $\left(\forall_{w \in(0 ; 1] \subset X}\left\{\lim _{m \rightarrow \infty} p\left(w^{m}, w\right)=0\right\}\right)$. Hence, by (75) and (76), we get $\forall_{w \in[2 ; 3) \cup(4 ; 6) \subset X}\left\{\lim _{m \rightarrow \infty} J\left(w, w^{m}\right)=\right.$ $0\}\left(\forall_{w \in(0 ; 1] \subset X}\left\{\lim _{m \rightarrow \infty} J\left(w^{m}, w\right)=0\right\}\right)$.

(5) $(X, T)$ is a left and right $\mathscr{P}_{\{8\} ;\{1\}}$-closed on $X$. Indeed, let $\left(x_{m}: m \in \mathbb{N}\right) \subset T(X)$ be a left (right) $\mathscr{P}_{\{8\} ;\{1\}}$-converging sequence in $X$ (thus $\left.\operatorname{LIM}_{\left(x_{m}: m \in \mathbb{N}\right)}^{L-\mathscr{D}_{\{8 ;: 1\}}} \neq \varnothing\left(\operatorname{LIM}_{\left(x_{m}: m \in \mathbb{N}\right)}^{R-\mathscr{P}_{\{8\}:\{1\}}} \neq \varnothing\right)\right)$ and having subsequences $\left(v_{m}: m \in \mathbb{N}\right)$ and $\left(u_{m}: m \in \mathbb{N}\right)$ satisfying $\forall_{m \in \mathbb{N}}\left\{v_{m} \in T\left(u_{m}\right)\right\}$. Then $\forall_{m \geq 2}\left\{x_{m} \in[1 ; 2]\right\}, 2 \in$

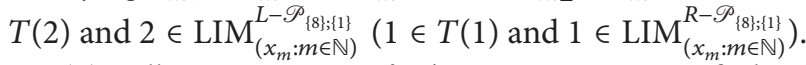

(6) All assumptions of Theorem 26 are satisfied. This follows from (1)-(5) in Example 1.

We conclude that $\operatorname{Fix}(T)=[1 ; 2]$ and we have shown the following.

Claim A. $2 \in T(2)$ and $2 \in \operatorname{LIM}_{\left(w^{m}: m \in\{0\} \cup \mathbb{N}\right)}^{L-\mathscr{P}_{\{8 ;:\{1\}}}$ for each $w^{0} \in X$ and for each dynamic process $\left(w^{m}: m \in\{0\} \cup \mathbb{N}\right)$ of the system $(X, T)$.

Claim B. $1 \in T(1)$ and $1 \in \operatorname{LIM}_{\left(w^{m}: m \in\{0\} \cup \mathbb{N}\right)}^{R-\mathscr{P}_{88: 11\}}}$ for each $w^{0} \in X$ and for each dynamic process $\left(w^{m}: m \in\{0\} \cup \mathbb{N}\right)$ of the $\operatorname{system}(X, T)$.

Example 2. Let $X, \mathscr{P}_{\{8\} ;\{1\}}=\{p\}$, and $(X, T)$ be such as in Example 1.

(1) For each $\lambda \in[0 ; 1)$, condition $\forall_{x, y \in X}\{8 D(T(x)$, $T(y)) \leq \lambda p(x, y)\}$, where $D(U, W)=\max \left\{\sup _{u \in U} p(u\right.$, $\left.W), \sup _{w \in W} p(U, w)\right\}, U, W \in 2^{X}$, does not hold. Suppose that $\exists_{\lambda_{0} \in[0 ; 1)} \forall_{x, y \in X}\left\{8 D(T(x), T(y)) \leq \lambda_{0} p(x, y)\right\}$. Letting $x_{0}=2$ and $y_{0}=3$, it can be shown that $p\left(x_{0}, y_{0}\right)=1, T\left(x_{0}\right)=$ $[1 ; 2]=U, T\left(y_{0}\right)=(4 ; 6)=W, \sup _{u \in[1 ; 2]} p(u,(4 ; 6))=$ $\sup _{u \in[1 ; 2]}(4-u)^{4}=3^{4}=81$, and $\sup _{w \in(4 ; 6)} p([1 ; 2], w)=$ $\sup _{w \in(4 ; 6)}(w-2)^{4}=4^{4}=256$. Therefore $2048=8 D\left(T\left(x_{0}\right)\right.$, $\left.T\left(y_{0}\right)\right)=8 \max \{81 ; 256\} \leq \lambda_{0} p\left(x_{0}, y_{0}\right)=\lambda_{0}$, which is absurd.

Remark 34. We make the following remarks about Examples 1 and 2 and Theorem 26: (a) By Example 1, we observe that we may apply Theorem 26 for set-valued dynamic systems $(X, T)$ in the left and right quasi-triangular space $\left(X, \mathscr{P}_{C ; \mathscr{A}}\right)$ with left and right family $\mathscr{J}_{C ; \mathscr{A}}$ generated by $\mathscr{P}_{C ; \mathscr{A}}$ where $\mathscr{J}_{\mathrm{C} ; \mathscr{A}} \neq \mathscr{P}_{\mathrm{C} ; \mathscr{A} \cdot}$ (b) By Example 2, we note, however, that we do not apply Theorem 26 in the quasi-triangular space $\left(X, \mathscr{P}_{C ; \mathscr{A}}\right)$ when $\mathscr{f}_{C ; \mathscr{A}}=\mathscr{P}_{C ; \mathscr{A} \text {. }}$ (c) From (a) and (b) it follows that, in Theorem 26, the existence of left (right) families $\mathscr{F}_{C ; A}$ generated by $\mathscr{P}_{C ; \mathscr{A}}$ and such that $\mathscr{J}_{C ; \mathscr{A}} \neq \mathscr{P}_{C ; \mathscr{A}}$ are essential. 
Example 3. Let $X=(0 ; 6), \gamma>0$, and

$$
A=A_{1} \cup A_{2}, \quad A_{1}=(0 ; 2], A_{2}=[4 ; 6) \text {. }
$$

Let $p: X^{2} \rightarrow[0 ; \infty)$ be of the form

$$
p(u, w)= \begin{cases}0 & \text { if } A \cap\{u, w\}=\{u, w\} \\ \gamma & \text { if } A \cap\{u, w\} \neq\{u, w\}\end{cases}
$$

and let $\mathscr{J}_{\{1\} ;\{1\}}=\mathscr{P}_{\{1\} ;\{1\}}=\{p\}$. Define the set-valued dynamic $\operatorname{system}(X, T)$ by

$$
T(u)= \begin{cases}A_{2} & \text { for } u \in(0 ; 3) \\ A & \text { for } u=3 \\ A_{1} & \text { for } u \in(3 ; 6)\end{cases}
$$

(1) $\left(X, \mathscr{P}_{\{1\} ;\{1\}}\right)$ is quasi-triangular space. See Example 2, Section 11.

(2) $(X, T)$ is a $\left(\mathscr{D}_{1 ; 2^{X}}^{L-\mathscr{P}_{\{1\} ; 1\}}}, \lambda \in[0 ; 1)\right)$-quasi-contraction on $X$; that is, $\forall_{\lambda \in[0 ; 1)} \forall_{x, y \in X}\left\{D_{1 ; 2^{X}}^{L-\mathscr{P}_{\{1\} ; 1\}}}(T(x), T(y)) \leq \lambda p(x\right.$, $y)\}$. Indeed, if $x, y \in X$, then, by (77)-(79), $T(x), T(y) \subset A$ and $\max \left\{\sup _{u \in T(x)} p(u, T(y)), \sup _{w \in T(y)} p(T(x), w)\right\}=0$.

(3) Property (16) holds; that is, $\forall_{x \in X} \forall_{\beta \in(0 ; \infty)}$ $\exists_{y \in T(x)}\{p(x, y)<p(x, T(x))+\beta\}$. Indeed, this follows from Cases 1-3 below.

Case 1. Let $x_{0} \in(0 ; 3)$ and $\beta \in(0 ; \infty)$ be arbitrary and fixed. If $y_{0} \in T\left(x_{0}\right)=A_{2}$, then, by (78),

$$
\begin{aligned}
p\left(x_{0}, y_{0}\right) & =p\left(x_{0}, T\left(x_{0}\right)\right) \\
& = \begin{cases}0 & \text { if } x_{0} \in A_{1}, \\
\gamma & \text { for } x_{0} \in(0 ; 3) \backslash A_{1} .\end{cases}
\end{aligned}
$$

Therefore, $p\left(x_{0}, y_{0}\right)<p\left(x_{0}, T\left(x_{0}\right)\right)+\beta$.

Case 2. Let $x_{0}=3$ and let $\beta \in(0 ; \infty)$ be arbitrary and fixed. If $y_{0} \in T\left(x_{0}\right)=A$, then, by (78), $p\left(x_{0}, y_{0}\right)=p\left(x_{0}, T\left(x_{0}\right)\right)=\gamma$. Therefore, $p\left(x_{0}, y_{0}\right)<p\left(x_{0}, T\left(x_{0}\right)\right)+\beta$.

Case 3. Let $x_{0} \in(3 ; 6)$ and $\beta \in(0 ; \infty)$ be arbitrary and fixed. If $y_{0} \in T\left(x_{0}\right)=A_{1}$, then, by (78),

$$
\begin{aligned}
p\left(x_{0}, y_{0}\right) & =p\left(x_{0}, T\left(x_{0}\right)\right) \\
& = \begin{cases}0 & \text { if } x_{0} \in A_{2}, \\
\gamma & \text { for } x_{0} \in(3 ; 6) \backslash A_{2} .\end{cases}
\end{aligned}
$$

Therefore, $p\left(x_{0}, y_{0}\right)<p\left(x_{0}, T\left(x_{0}\right)\right)+\beta$.

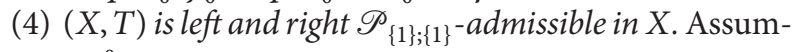
ing that $w^{0} \in X$ is arbitrary and fixed we prove that if the dynamic process $\left(w^{m}: m \in\{0\} \cup \mathbb{N}\right)$ of $(X, T)$ starting at $w^{0}$ is such that $\lim _{m \rightarrow \infty} \sup _{n>m} p\left(w^{m}, w^{n}\right)=0$, then $\exists_{w \in X}\left\{\lim _{m \rightarrow \infty} p\left(w, w^{m}\right)=0\right\}$. Indeed, if $w^{0} \in X$, then, by
(79), $\forall_{m \geqslant 1}\left\{w^{m} \in T\left(w^{m-1}\right) \subset A\right\}$ and, by (78), we immediately get $A=\operatorname{LIM}_{\left(w^{m}: m \in\{0\} \cup \mathbb{N}\right)}^{L-\mathscr{P}_{\{1\} ; 1\}}}=\operatorname{LIM}_{\left(w^{m}: m \in\{0\} \cup \mathbb{N}\right)}^{R-\mathscr{P}_{\{1\} ; 1\}}}$.

(5) Set-valued dynamic system $\left(X, T^{[2]}\right)$ is a left and right

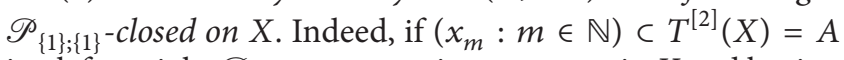

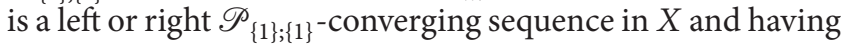
subsequences $\left(v_{m}: m \in \mathbb{N}\right)$ and $\left(u_{m}: m \in \mathbb{N}\right)$ satisfying $\forall_{m \in \mathbb{N}}\left\{v_{m} \in T\left(u_{m}\right)\right\}$, then, by (77)-(79), we have that $\exists_{m_{0} \in \mathbb{N}} \forall_{m \geqslant m_{0}}\left\{x_{m} \in A\right\}, A=\operatorname{LIM}_{\left(x_{m}: m \in \mathbb{N}\right)}^{L-\mathscr{P}_{\{1\} ; 1\}}}=\operatorname{LIM}_{\left(x_{m}: m \in\{0\} \cup \mathbb{N}\right)}^{R-\mathscr{P}_{\{1\} ; 1\}}}$, and $\operatorname{Fix}\left(T^{[2]}\right)=A$.

(6) For $\left(X, \mathscr{P}_{\{1\} ;\{1\}}\right), \mathscr{P}_{\{1\} ;\{1\}}=\{p\}, \mathscr{J}_{\{1\} ;\{1\}}=\mathscr{P}_{\{1\} ;\{1\}}$, and $(X, T)$ defined by (77)-(79), all assumptions of Theorem 26 are satisfied. This follows from (1)-(5) in Example 3.

We conclude that $\operatorname{Fix}\left(T^{[2]}\right)=A$ and we claim that if $w^{0} \epsilon$ $X, w^{1} \in T\left(w^{0}\right)$, and $w^{2}=u \in T\left(w^{1}\right)$ are arbitrary and fixed, and $\forall_{m \geqslant 3}\left\{w^{m}=u\right\}$, then sequence $\left(w^{m}: m \in\{0\} \cup \mathbb{N}\right)$ is a dynamic process of $T$ starting at $w^{0}$ and left and right $\mathscr{P}_{\{1\} ;\{1\}^{-}}$ converging to each point of $A$. We observe also that $\operatorname{Fix}(T)=$ $\varnothing$.

Example 4. Let $X=[0 ; 6]$ and let $\mathscr{P}_{\{2\} ;\{1\}}=\{p\}$ where $p$ : $X^{2} \rightarrow[0 ; \infty)$ is of the form

$$
p(u, w)= \begin{cases}0 & \text { if } u \geq w \\ (u-w)^{2} & \text { if } u<w .\end{cases}
$$

Define the set-valued dynamic system $(X, T)$ by

$$
T(u)=([0 ; 3) \cup(3 ; 6]) \backslash\{u\} \quad \text { for } u \in[0 ; 6] \text {. }
$$

Let

$$
E=[0 ; 3) \cup(3 ; 6]
$$

and let $\mu \geq 36 / 4$ and $\mathscr{J}_{\{2\} ;\{1\}}=\{J\}$ where $J: X^{2} \rightarrow[0 ; \infty)$ is of the form

$$
J(u, w)= \begin{cases}p(u, w) & \text { if } E \cap\{u, w\}=\{u, w\} \\ \mu & \text { if } E \cap\{u, w\} \neq\{u, w\}\end{cases}
$$

(1) $\mathscr{J}_{\{2\} ;\{1\}}$ is not symmetric. In fact, by (82), (84), and (85), $J(0,6)=36$ and $J(6,0)=0$.

(2) $\mathscr{J}_{\{2\} ;\{1\}}=\{J\} \in \mathbb{J}_{\left(X, \mathscr{P}_{\{2\} ;\{1\}}\right)}^{L} \cap \mathbb{J}_{\left(X, \mathscr{P}_{\{2\} ; 1\}}\right)}^{R}$. See Theorem 14.

(3) $(X, T)$ is a $\left(D=\mathscr{D}_{1 ; 2^{X}}^{L-\mathscr{J}_{\{2\} ; 1\}}}, \lambda \in[0 ; 1)\right)$-contraction on $X$; that is, $\forall_{x, y \in X}\{2 \cdot D(T(x), T(y)) \leq \lambda J(x, y)\}$ where $\lambda \epsilon$ $[0 ; 1)$ and

$$
\begin{array}{r}
D(U, W)=\max \left\{\sup _{u \in U}(u, W), \sup _{w \in W} J(U, w)\right\}, \\
\qquad, W \in 2^{X} .
\end{array}
$$

Indeed, we see that this follows from (1), (2) in Example 4, and from Cases 1-4 below. 
Case 1. Let $x, y \in[0 ; 3) \cup(3 ; 6]$. Then $x, y \in E, T(x)=([0 ; 3) \cup$ $(3 ; 6]) \backslash\{x\}=U \subset E$, and $T(y)=([0 ; 3) \cup(3 ; 6]) \backslash\{y\}=W \subset E$. If $u \in U$, then we have $W=W^{u} \cup W_{u}$ and

$$
\begin{aligned}
& \inf _{w \in W} J(u, w) \\
& \leq \begin{cases}\inf _{w \in W^{u}} q(u, w)=0 & \text { if } W^{u}=\{w \in W: u \geq w\} \neq \varnothing, \\
\inf _{w \in W_{u}}(u-w)^{2}=0 & \text { if } W_{u}=\{w \in W: u<w\} \neq \varnothing\end{cases}
\end{aligned}
$$

and if $w \in W$, then we have $U=U^{w} \cup U_{w}$ and

$$
\begin{aligned}
& \inf _{u \in U} J(u, w) \\
& \leq\left\{\begin{array}{cl}
\inf _{u \in U^{w}} q(u, w)=0 & \text { if } U^{u}=\{u \in U: u \geq w\} \neq \varnothing, \\
\inf _{u \in U_{w}}(u-w)^{2}=0 & \text { if } U_{u}=\{u \in U: u<w\} \neq \varnothing .
\end{array}\right.
\end{aligned}
$$

By $(86), 2 D(T(x), T(y))=0 \leq \lambda J(x, y)$.

Case 2. If $x=y=3$, then $J(x, y)=\mu$ and $T(x)=$ $T(y)=[0 ; 3) \cup(3 ; 6]=U \subset E$. Therefore, $2 D(T(x), T(y))=$ $2 D(U, U)=0 \leq \lambda J(x, y)$.

Case 3. If $x \in[0 ; 3) \cup(3 ; 6]$ and $y=3$, then $x \in E, y \notin E$, $J(x, y)=\mu, T(x)=([0 ; 3) \cup(3 ; 6]) \backslash\{x\}=U \subset E$, and $T(y)=$ $[0 ; 3) \cup(3 ; 6]=W \subset E$. We see that $\sup _{u \in U}\left\{\inf _{w \in W} J(u, w)\right\}=$ 0 since if $u \in U$, then also $w=u \in W$ and $\inf _{w \in W} J(u, w)=$ $q(u, u)=0$. Next, we see that $\sup _{w \in W}\left\{\inf _{u \in U} J(u, w)\right\}=0$ since if $w \in W$, then $U=U^{w} \cup U_{w}$ and

$$
\begin{aligned}
& \inf _{u \in U} J(u, w) \\
& \leq \begin{cases}\inf _{u \in U^{w}} q(u, w)=0 & \text { if } U^{u}=\{u \in U: u \geq w\} \neq \varnothing, \\
\inf _{u \in U_{w}}(u-w)^{2}=0 & \text { if } U_{u}=\{u \in U: u<w\} \neq \varnothing .\end{cases}
\end{aligned}
$$

Thus $2 D(T(x), T(y))=0 \leqslant \lambda J(x, y)$.

Case 4. If $x=3$ and $y \in[0 ; 3) \cup(3 ; 6]$, then $x \notin E, y \in E$, $J(x, y)=\mu, T(x)=[0 ; 3) \cup(3 ; 6]=U \subset E, T(y)=([0 ; 3) \cup$ $(3 ; 6]) \backslash\{y\}=W \subset E$, and $\sup _{u \in U}\left\{\inf _{w \in W} J(u, w)\right\}=0$ since, for $u \in U$,

$$
\begin{aligned}
& \inf _{w \in W} J(u, w) \\
& \leq \begin{cases}\inf _{w \in W^{u}} q(u, w)=0 & \text { if } W^{u}=\{w \in W: u \geq w\} \neq \varnothing, \\
\inf _{w \in W_{u}}(u-w)^{2}=0 & \text { if } W_{u}=\{w \in W: u<w\} \neq \varnothing\end{cases}
\end{aligned}
$$

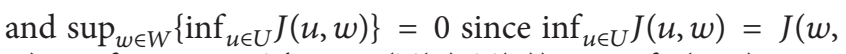
$w)=0$ for $w \in W$. Thus $2 D(T(x), T(y))=0 \leq \lambda J(x, y)$.

(4) Property (26) holds; that is, $\forall_{x \in X} \forall_{\gamma \in(0 ; \infty)} \exists_{y \in T(x)}\{J(x$, $y)<J(x, T(x))+\gamma\}$. Indeed, this follows from Cases 1-3 below.

Case 1. Let $x_{0} \in[0 ; 3)$ and $\gamma \in(0 ; \infty)$ be arbitrary and fixed. If $y_{0} \in T\left(x_{0}\right)=([0 ; 3) \cup(3 ; 6]) \backslash\left\{x_{0}\right\}=W$ is such that $x_{0}<y_{0}<3$, then $J\left(x_{0}, y_{0}\right)=\left(x_{0}-y_{0}\right)^{2}$ and $J\left(x_{0}, T\left(x_{0}\right)\right)=$ $\inf _{w \in W} J\left(x_{0}, w\right)=0$ since

$$
\begin{aligned}
& \inf _{w \in W} J\left(x_{0}, w\right) \\
& \leq \begin{cases}\inf _{w \in W^{x_{0}}} q\left(x_{0}, w\right)=0 & \text { if } W^{x_{0}}=\left\{w \in W: x_{0} \geq w\right\} \neq \varnothing, \\
\inf _{w \in W_{x_{0}}}\left(x_{0}-w\right)^{2}=0 & \text { if } W_{x_{0}}=\left\{w \in W: x_{0}<w\right\} \neq \varnothing .\end{cases}
\end{aligned}
$$

Then we see that $J\left(x_{0}, y_{0}\right)=\left(x_{0}-y_{0}\right)^{2}<\gamma$ implies $y_{0}<x_{0}+$ $\gamma^{1 / 2}$. From this we conclude that if $y_{0} \in\left(x_{0} ; \min \left\{3, x_{0}+\gamma^{1 / 2}\right\}\right)$, then $J\left(x_{0}, y_{0}\right)<J\left(x_{0}, T\left(x_{0}\right)\right)+\gamma$.

Case 2. Let $x_{0}=3$. Assume that $y_{0} \in T\left(x_{0}\right)=[0 ; 3) \cup(3 ; 6]$ is arbitrary and fixed. Then $J\left(x_{0}, y_{0}\right)=\mu, J\left(x_{0}, T\left(x_{0}\right)\right)=$ $\inf _{w \in[0 ; 3) \cup(3 ; 6]} J\left(x_{0}, w\right)=\mu$ and, for each $\gamma \in(0 ; \infty)$, $J\left(x_{0}, y_{0}\right)<J\left(x_{0}, T\left(x_{0}\right)\right)+\gamma$.

Case 3. Let $x_{0} \in(3 ; 6]$ and $\gamma \in(0 ; \infty)$ be arbitrary and fixed. If $y_{0} \in T\left(x_{0}\right)=([0 ; 3) \cup(3 ; 6]) \backslash\left\{x_{0}\right\}=W$ is such that $3<$ $y_{0}<x_{0}$, then $J\left(x_{0}, y_{0}\right)=0$ and, analogously as in Case 1 , we get $J\left(x_{0}, T\left(x_{0}\right)\right)=\inf _{w \in W} J\left(x_{0}, w\right)=0$. Therefore, $J\left(x_{0}, y_{0}\right)<$ $J\left(x_{0}, T\left(x_{0}\right)\right)+\gamma$.

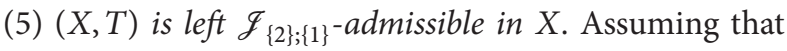
$w^{0} \in X$ is arbitrary and fixed we prove that if the dynamic process $\left(w^{m}: m \in\{0\} \cup \mathbb{N}\right)$ of $(X, T)$ starting at $w^{0}$ is such that $\lim _{m \rightarrow \infty} \sup _{n>m} J\left(w^{m}, w^{n}\right)=0$, then $\exists_{w \in X}\left\{\lim _{m \rightarrow \infty} J\left(w, w^{m}\right)=0\right\}$. We consider the following cases.

Case 1. If $w^{0} \in[0 ; 3) \cup(3 ; 6]$, then $w^{1} \in T\left(w^{0}\right)=([0 ; 3) \cup$ $(3 ; 6]) \backslash\left\{w^{0}\right\}$ and $\forall_{m \geq 2}\left\{w^{m} \in T\left(w^{m-1}\right) \subset[0 ; 3) \cup(3 ; 6]\right\}$ and using (82) we immediately get $6 \in \operatorname{LIM}_{\left(w^{m}: m \in\{0\} \cup \mathbb{N}\right)}^{L-\mathcal{F}_{\{2\} ; 1\}}}$.

Case 2. If $w^{0}=3$, then $w^{1} \in T\left(w^{0}\right)=[0 ; 3) \cup(3 ; 6], w^{2} \in$ $T\left(w^{1}\right)=([0 ; 3) \cup(3 ; 6]) \backslash\left\{w^{1}\right\}$, and $\forall_{m \geqslant 3}\left\{w^{m} \in T\left(w^{m-1}\right) \subset\right.$ $[0 ; 3) \cup(3 ; 6]\}$ and using $(82)$ we also immediately get $6 \in$ $\operatorname{LIM}_{\left(w^{m}: m \in\{0\} \cup \mathbb{N}\right)}^{L-\mathcal{F}_{\{2\} ;\{\}}}$.

This shows that $6 \in \operatorname{LIM}_{\left(w^{m}: m \in\{0\} \cup \mathbb{N}\right)}^{L-\mathcal{F}_{\{2\} ; 1\}}}$ for each $w^{0} \in X$ and for each dynamic process $\left(w^{m}: m \in\{0\} \cup \mathbb{N}\right)$ of the system $(X, T)$; we see that here property $\lim _{m \rightarrow \infty} \sup _{n>m} J\left(w^{m}, w^{n}\right)=$ 0 of $\left(w^{m}: m \in\{0\} \cup \mathbb{N}\right)$ is not required.

(6) Set-valued dynamic system $\left(X, T^{[2]}\right)$ is a left $\mathscr{P}_{\{2\} ;\{1\}^{-}}$ quasi-closed on $X$. Indeed, if $\left(x_{m}: m \in \mathbb{N}\right) \subset T^{[2]}(X)=$ $[0 ; 3) \cup(3 ; 6]$ is a left $\mathscr{P}_{\{2\} ;\{1\}}$-converging sequence in $X$ and having subsequences $\left(v_{m}: m \in \mathbb{N}\right)$ and $\left(u_{m}: m \in \mathbb{N}\right)$ satisfying $\forall_{m \in \mathbb{N}}\left\{v_{m} \in T\left(u_{m}\right)\right\}$, then, by (83), we have that $\exists_{m_{0} \in \mathbb{N}} \forall_{m \geq m_{0}}\left\{x_{m} \in[0 ; 3) \cup(3 ; 6]\right\}$. Therefore, in particular, $6 \in \operatorname{LIM}_{\left(x_{m}: m \in \mathbb{N}\right)}^{L-\mathscr{P}_{\{2\} ;\{1\}}}$ and $6 \in T^{[2]}(6)$.

(7) For $\mathscr{P}_{\{2\} ;\{1\}}=\{p\}, \mathscr{J}_{\{2\} ;\{1\}}=\{J\}$, and $(X, T)$ defined by (82)-(85), all assumptions of Theorem 26 in the case of "left" are satisfied. This follows from (1)-(6) in Example 4.

We conclude that $\operatorname{Fix}\left(T^{[2]}\right)=[0 ; 3) \cup(3 ; 6]$ and we claim that $6 \in T^{[2]}(6)$ and that $6 \in \operatorname{LIM}_{\left(w^{m}: m \in\{0\} \cup \mathbb{N}\right)}^{L-\mathscr{P}_{\{2\} ; 1\}}}$ for each $w^{0} \in X$ 
and for each dynamic process $\left(w^{m}: m \in\{0\} \cup \mathbb{N}\right)$ of the system $(X, T)$. We observe also that $\operatorname{Fix}(T)=\varnothing$.

\section{Example Illustrating Theorem 32}

Example 1. Let $X=(0 ; 6), A$, and $\mathscr{F}_{\{1\} ;\{1\}}=\mathscr{P}_{\{1\} ;\{1\}}=\{p\}$ be as in Example 3. Define the single-valued dynamic system $(X, T)$ by

$$
T(u)= \begin{cases}4 & \text { for } u \in(0 ; 3), \\ 2 & \text { for } u \in[3 ; 6) .\end{cases}
$$

(1) $(X, T)$ is a $\left(\mathscr{D}_{1 ; X}^{L-\mathscr{P}_{\{1 ; ; 1\}}}, \lambda \in[0 ; 1)\right)$-quasi-contraction on $X$; that is, $\forall_{\lambda \in[0 ; 1)} \forall_{x, y \in X}\left\{D_{1 ; X}^{L-\mathscr{P}_{\{1 ; ; 1\}}}(T(x), T(y))=\right.$ $\max \{p(T(x), T(y)), p(T(y), T(x))\} \leq \lambda p(x, y)\}$ and $\operatorname{Fix}(T)=$ $\varnothing$. Indeed, we see that if $x, y \in X$, then $T(x), T(y) \in A$ and, by (77) and (78), $D_{1 ; X}^{L-\mathscr{P}_{\{1 ; ; 1\}}}(T(x), T(y))=0 \leq \lambda p(x, y)$.

(2) $(X, T)$ is left and right $\mathscr{P}_{\{1\} ;\{1\} \text {-admissible in } X \text {. Assume }}$ that $w^{0} \in X$ is arbitrary and fixed, $\left(w^{m}: m \in\{0\} \cup \mathbb{N}\right)$ satisfies $\forall_{m \in\{0\} \cup \mathbb{N}}\left\{w^{m+1}=T\left(w^{m}\right)\right\}$, and $\lim _{m \rightarrow \infty} \sup _{n>m} p\left(w^{m}, w^{n}\right)=$ 0 . Then, by (92) and (78), we have $\forall_{m \in \mathbb{N}}\left\{w^{m} \in A\right\}$. This gives $A=\operatorname{LIM}_{\left(w^{m}: m \in\{0\} \cup \mathbb{N}\right)}^{L-\mathscr{P}_{\{1\} ; 1\}}}=\operatorname{LIM}_{\left(w^{m}: m \in\{0\} \cup N\right)}^{R-\mathscr{P}_{\{1\} 1\}}}$.

(3) Single-valued dynamic system $\left(X, T^{[2]}\right)$ is a left and right $\mathscr{P}_{\{1\} ;\{1\}}$-closed on $X$. Indeed, if $\left(x_{m}: m \in \mathbb{N}\right) \subset$

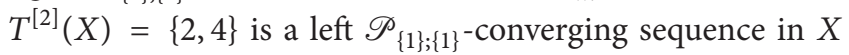
and having subsequences $\left(v_{m}: m \in \mathbb{N}\right)$ and $\left(u_{m}: m \in \mathbb{N}\right)$ satisfying $\forall_{m \in \mathbb{N}}\left\{v_{m}=T^{[2]}\left(u_{m}\right)\right\}$, then, by (77), (78), and (92),

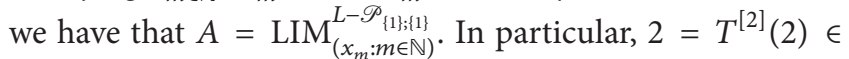
$\operatorname{LIM}_{\left(x_{m}: m \in \mathbb{N}\right)}^{L-\mathscr{P}_{\{1 ; 11\}}}$ and $4=T^{[2]}(4) \in \operatorname{LIM}_{\left(x_{m}: m \in \mathbb{N}\right)}^{L-\mathscr{P}_{\{1 \mid ; 1\}}}$.

(4) Property (56) holds. Indeed, we have $\forall_{v \in \operatorname{Fix}\left(T^{[2]}\right)=\{2,4\}}$ $\{p(v, T(v))=p(T(v), v)=0\}$ since $T(2)=4, T(4)=2$, and $T(\{2,4\})=\{2,4\} \subset A$.

(5) $\mathscr{P}_{\{1\} ;\{1\}}=\{p\}$ is not separating on $X$. Indeed, if $u, w \in$ $X / A$, then $p(u, w)=p(w, u)=\gamma>0$.

(6) For $\mathscr{P}_{\{1\} ;\{1\}}=\{p\},(X, T)$, and $\mathscr{J}_{\{1\} ;\{1\}}=\mathscr{P}_{\{1\} ;\{1\}}$ defined by (77), (78), and (79) parts (B1) and (B2) of Theorem 32 hold but part (B3) of Theorem 32 does not hold. This follows from (1)-(5) in Example 1.

\section{Concluding Remarks}

Remark 1. In Theorems 5 and 6 the following play an important role: (i) Distances $d$ and $H^{d}$, as metrics, satisfy conditions (A) of Definition 1 on $X$ and $\mathscr{C} \mathscr{B}(X)$, respectively. (ii) $(X, d)$ and $\left(\mathscr{C} \mathscr{B}(X), H^{d}\right)$, as metric spaces, are topological and Hausdorff spaces and the completeness of $(X, d)$ implies completeness of $\left(\mathscr{C} \mathscr{B}(X), H^{d}\right)$. (iii) The continuity of $d$ and $H^{d}$ on $X \times X$ and $\mathscr{C} \mathscr{B}(X) \times \mathscr{C} \mathscr{B}(X)$, respectively; (iv) The continuity of maps $T:(X, d) \rightarrow(X, d)$ and $T:$ $(X, d) \rightarrow\left(\mathscr{C} \mathscr{B}(X), H^{d}\right)$ (as consequences of contractive properties defined in (1) and (3), resp.); (v) In Theorem 6 the assumption that, for each $x \in X, T(x) \in \mathscr{C} \mathscr{B}(X)$.

Remark 2. Conclusions in Theorems 5 and 6 concern only fixed points but not periodic points; this is a consequence of separability of spaces $(X, d)$ and $\left(\mathscr{C} \mathscr{B}(X), H^{d}\right)$ and also continuity of $T$.

Remark 3. In Theorems 26 and 32, properties concening the spaces and maps such as mentioned above generally need not hold, since spaces $\left(X, \mathscr{P}_{C ; \mathscr{A}}\right)$ with left (right) families $\mathscr{J}_{C ; \mathscr{A}}$ generated by $\mathscr{P}_{C ; \mathscr{A} A}$ are very general, which is an obstruction to use Nadler's and Banach's reasoning. Theorems 26 and 32 show how to rectify this situation and are obtained without restrictively required assumptions and with conclusions more profound as in the well known results of this sort existing in the literature.

\section{Conflict of Interests}

The author declares that he has no conflict of interests regarding the publication of this paper.

\section{References}

[1] J.-P. Aubin and J. Siegel, "Fixed points and stationary points of dissipative multivalued maps," Proceedings of the American Mathematical Society, vol. 78, no. 3, pp. 391-398, 1980.

[2] J. P. Aubin and I. Ekeland, Applied Nonlinear Analysis, John Wiley \& Sons, New York, NY, USA, 1984.

[3] J.-P. Aubin and H. Frankowska, Set-Valued Analysis, vol. 2 of Systems \& Control: Foundations \& Applications, Birkhäuser, Boston, Mass, USA, 1990.

[4] G. X.-Z. Yuan, KKM Theory and Applications in Nonlinear Analysis, Marcel Dekker, New York, NY, USA, 1999.

[5] A. C. van Roovij, Non Archimedean Functional Analysis, Marcel Dekker, New York, NY, USA, 1978.

[6] I. A. Bakhtin, "The contraction mapping principle in almost metric space," Functional Analysis. Ulyanovsk State Pedagogical University, Ulyanovsk, Russia, vol. 30, pp. 26-37, 1989.

[7] S. Czerwik, "Nonlinear set-valued contraction mappings in b-metric spaces," Atti del Seminario Matematico e Fisico dell'Università di Modena, vol. 46, pp. 263-276, 1998.

[8] S. G. Matthews, "Partial metric topology," Annals of the New York Academy of Sciences, vol. 726, pp. 183-197, 1994, Proceedings of the 8th Summer Conference on General Topology and Application.

[9] S. Shukla, "Partial b-metric spaces and fixed point theorems," Mediterranean Journal of Mathematics, vol. 11, no. 2, pp. 703711, 2014.

[10] W. A. Wilson, “On quasi-metric spaces," American Journal of Mathematics, vol. 53, no. 3, pp. 675-684, 1931.

[11] H.-P. A. Künzi and O. O. Otafudu, "The ultra-quasi-metrically injective hull of a $T_{0}$-ultra-quasi-metric space," Applied Categorical Structures, vol. 21, pp. 651-670, 2013.

[12] J. Dugundji, Topology, Allyn \& Bacon, Boston, Mass, USA, 1966.

[13] I. L. Reilly, "Quasi-gauge spaces," Journal of the London Mathematical Society. Second Series, vol. 6, pp. 481-487, 1973.

[14] S. Banach, "Sur les operations dans les ensembles abstraits et leurs applications aux equations integrales," Fundamenta Mathematicae, vol. 3, pp. 133-181, 1922.

[15] S. B. Nadler, "Multi-valued contraction mappings," Notices of the American Mathematical Society, vol. 14, p. 930, 1967.

[16] S. B. Nadler Jr., "Multi-valued contraction mappings," Pacific Journal of Mathematics, vol. 30, pp. 475-488, 1969. 
[17] M. M. Deza and E. Deza, Encyclopedia of Distances, Springer, Berlin, Germany, 2nd edition, 2013.

[18] W. A. Kirk and N. Shahzad, Fixed Point Theory in Distance Spaces, Springer, Berlin, Germany, 2014.

[19] J. T. Markin, "Fixed point theorems for set valued contractions," Notices of the American Mathematical Society, vol. 15, p. 930, 1968.

[20] J. T. Markin, "A fixed point theorem for set valued mappings," Bulletin of the American Mathematical Society, vol. 74, pp. 639640, 1968.

[21] V. Berinde and M. Pacurar, "The role of the Pompeiu-Hausdorff metric in fixed point theory," Creative Mathematics and Informatics, vol. 22, no. 2, pp. 143-150, 2013.

[22] D. Tataru, "Viscosity solutions of Hamilton-Jacobi equations with unbounded nonlinear terms," Journal of Mathematical Analysis and Applications, vol. 163, no. 2, pp. 345-392, 1992.

[23] O. Kada, T. Suzuki, and W. Takahashi, "Nonconvex minimization theorems and fixed point theorems in complete metric spaces," Mathematica Japonica, vol. 44, no. 2, pp. 381-391, 1996.

[24] L.-J. Lin and W.-S. Du, "Ekeland's variational principle, minimax theorems and existence of nonconvex equilibria in complete metric spaces," Journal of Mathematical Analysis and Applications, vol. 323, no. 1, pp. 360-370, 2006.

[25] T. Suzuki, "Generalized distance and existence theorems in complete metric spaces," Journal of Mathematical Analysis and Applications, vol. 253, no. 2, pp. 440-458, 2001.

[26] J. S. Ume, "Existence theorems for generalized distance on complete metric spaces," Fixed Point Theory and Applications, vol. 2010, Article ID 397150, 21 pages, 2010.

[27] I. Vályi, "A general maximality principle and a fixed point theorem in uniform space," Periodica Mathematica Hungarica, vol. 16, no. 2, pp. 127-134, 1985.

[28] K. Włodarczyk, "Hausdorff quasi-distances, periodic and fixed points for Nadler type set-valued contractions in quasi-gauge spaces," Fixed Point Theory and Applications, vol. 2014, no. 1, article 239, 2014.

[29] K. Wlodarczyk and R. Plebaniak, "Maximality principle and general results of Ekeland and Caristi types without lower semicontinuity assumptions in cone uniform spaces with generalized pseudodistances," Fixed Point Theory and Applications, vol. 2010, Article ID 175453, p. 35, 2010.

[30] K. Włodarczyk and R. Plebaniak, "Dynamic processes, fixed points, endpoints, asymmetric structures, and investigations related to Caristi, Nadler, and Banach in uniform spaces," Abstract and Applied Analysis, vol. 2015, Article ID 942814, 16 pages, 2015. 


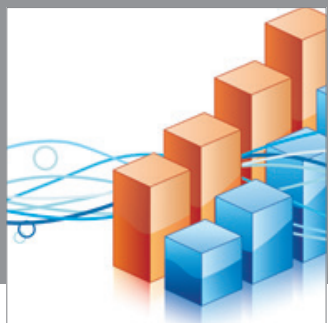

Advances in

Operations Research

mansans

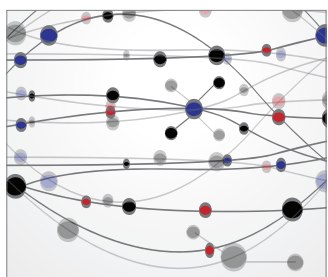

The Scientific World Journal
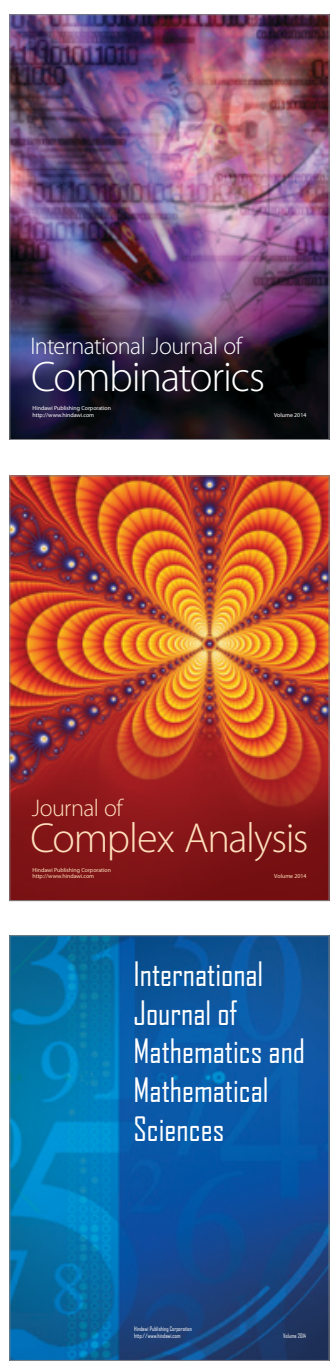
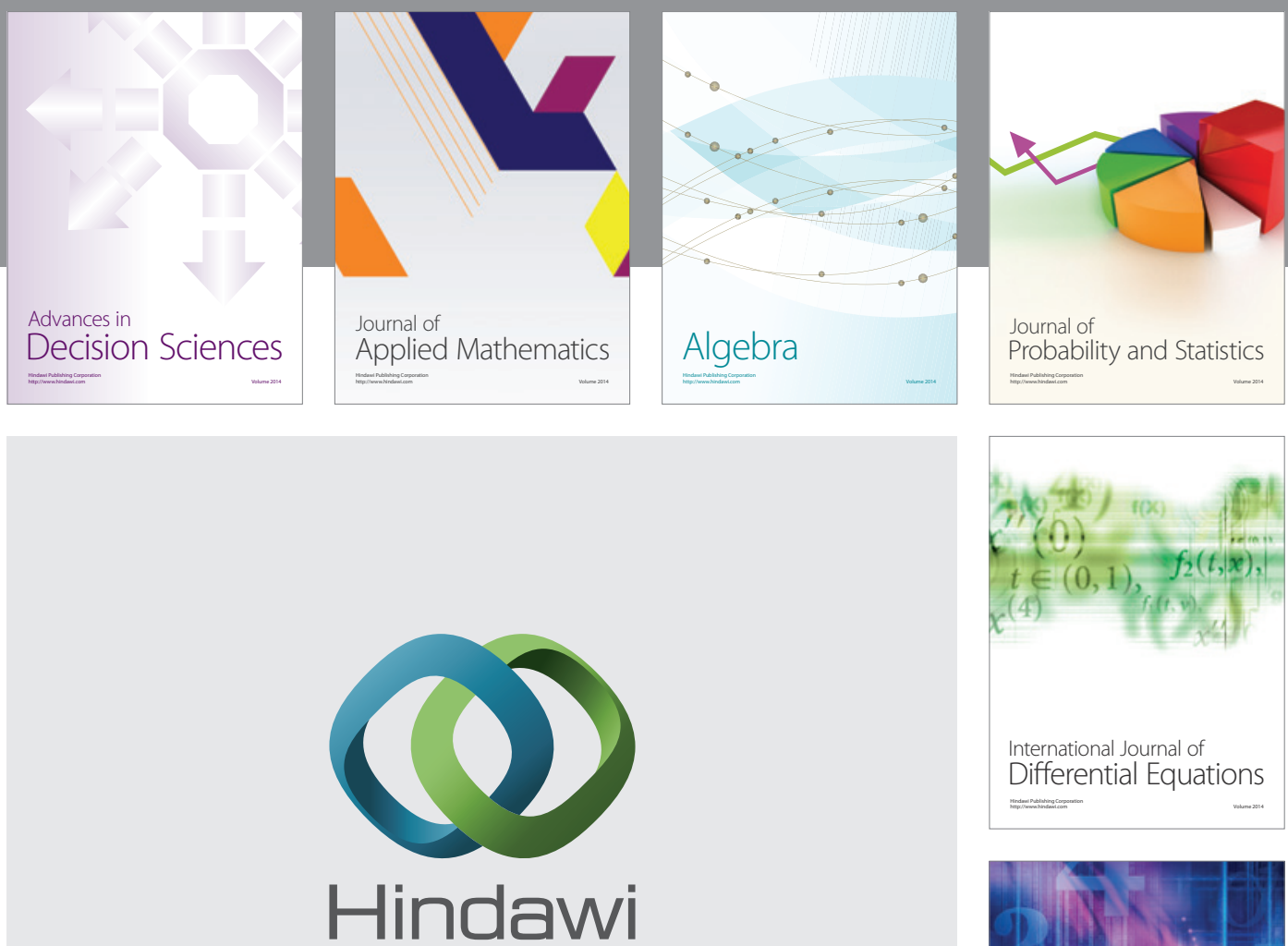

Submit your manuscripts at http://www.hindawi.com
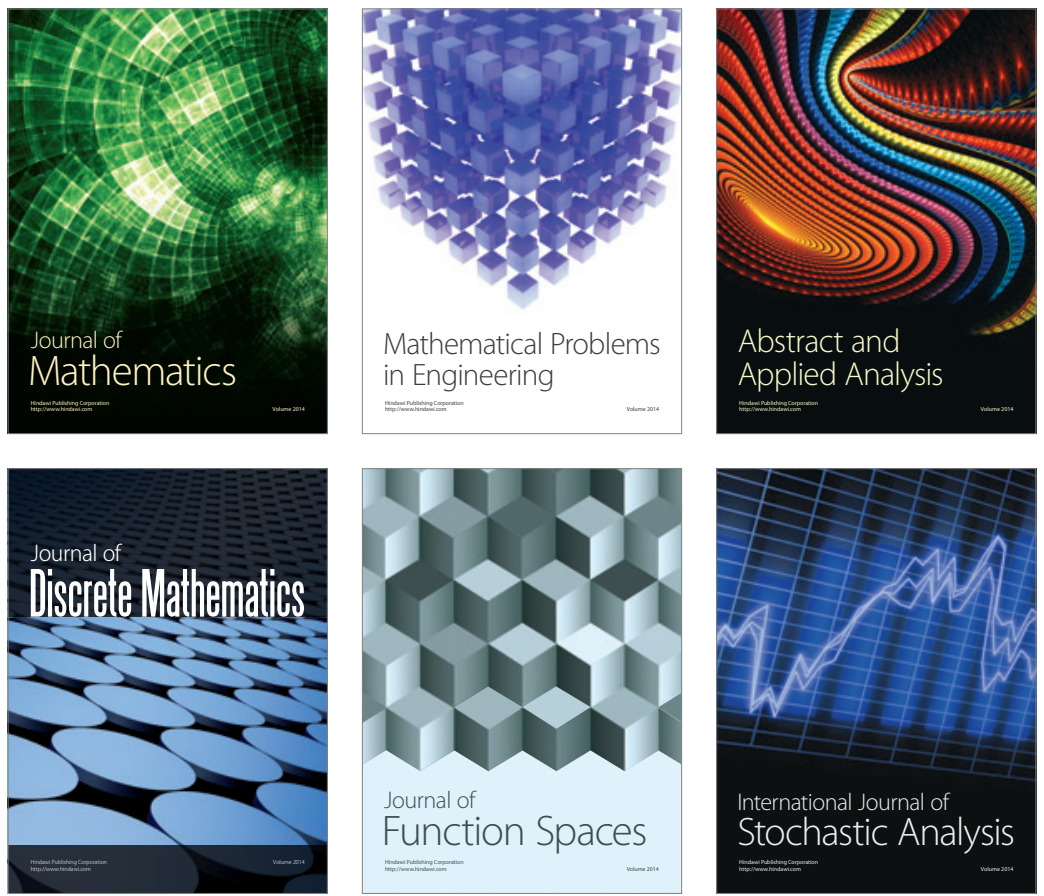

Journal of

Function Spaces

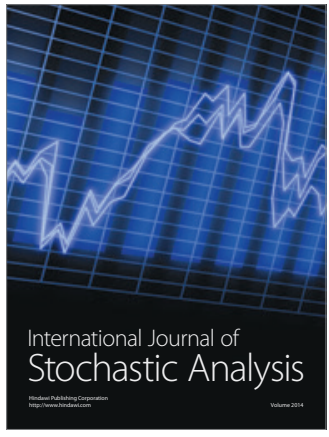

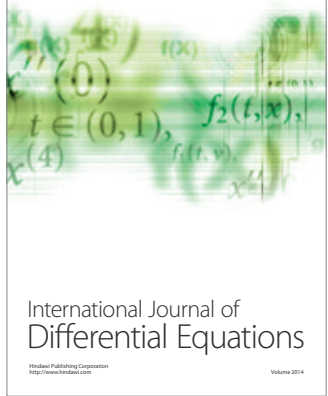
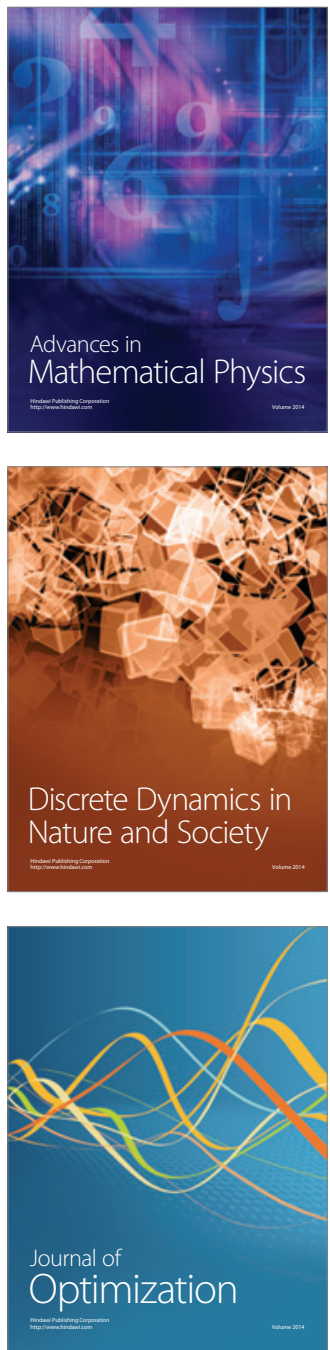